\title{
Settled and unsettled issues in particle settling
}

\author{
Roberto Piazza \\ Department of Chemistry, Material Science, and Chemical Engineering Politecnico di Milano, via Ponzio 34/3, 20133 Milano, Italy \\ E-mail: roberto.piazza@polimi.it
}

Received 19 November 2013, revised 18 February 2014

Accepted for publication 26 March 2014

Published 6 May 2014

Invited by Paul Chaikin

\begin{abstract}
Colloid sedimentation has played a seminal role in the development of statistical physics thanks to the celebrated experiments by Perrin, which provided a concrete demonstration of molecular reality and gave strong support to Einstein's theory of Brownian motion. This review, which mostly focuses on settling at low Peclét number, where Brownian fluctuations are dominant, aims to show that a lot more can be learnt both from the sedimentation equilibrium and from the particle settling dynamics of a wide class of systems, ranging from simple colloids to mesogenic suspensions, from soft solids to active particles and living organisms. At the same time, the occurrence of unexpected and surprising effects brings about challenging questions in statistical and fluid mechanics that make sedimentation an exciting field of research.
\end{abstract}

Keywords: sedimentation, colloids, particulate matter

(Some figures may appear in colour only in the online journal)

\section{Prelude: the perfect yellow}

Sedimentation, the progressive deposition of particulate settling in a fluid, is widespread in the natural environment. For example, it begets majestic depositional landforms like the Dolomites, provides valuable energy supplies such as vast oil shales [1], affects airborne particle pollution [2], and controls the distribution of plankton in the oceans [3]. This review, however, is neither concerned with the physical aspects of natural sedimentation in geology, ecology, and environmental science, nor with the countless applications of forced sedimentation (centrifugation) as a separation tool in the extractive, chemical, nuclear, and food processing industry [4], nor as a preparative and analytic method in biology and medicine [5]. Rather, I will just highlight those physical issues in settling phenomena that may provide basic insights to those scientists who are not specifically dealing with soft matter or fluid dynamics. As a matter of fact, sedimentation has played an important role in the development of statistical mechanics since its dawn; starting with a brief historical introduction is therefore appropriate.

Garcinia morella is a tree growing in India and Sri Lanka that, when tapped, supplies Indian gamboge, a saffroncolored resin traditionally considered by the Theravāda Buddhist monks as the 'perfect yellow' pigment for their robes. Gamboge, which is actually a natural water emulsion, proved to be a perfect material too for the landmark experimental investigation performed in 1908 by Jean Baptiste Perrin, which in fact turned colloids from a subject of interest for chemists to a benchmark test for Einstein's theory of Brownian motion and, more generally, for the molecular theory of matter. By studying the concentration profile that settling induces in suspensions of gamboge droplets $^{1}$ and comparing them to the barometric density profile that sets in ideal gases at isothermal

\footnotetext{
1 Subsequently, Perrin also studied emulsions made of mastic, another plant resin already known by the Greeks as 'the tears of Chios' and surely tasted by anybody who ever masticated a chewing gum, as the etymology of this verb suggests.
} 
equilibrium under gravity, Perrin was actually able to obtain an impressive series of successes, ranging from the determination of Avogadro's number and of the electron charge, to the experimental confirmation of the law of equipartition of energy, which are lucidly summarized in [6]. Reading this work, a masterpiece of experimental ingenuity, unravels several other details that are by no means marginal. For instance, the accurate historical discussion presented in the first part of the paper makes it clear that Perrin was fully conscious of the key importance for statistical physics of van't Hoff law for dilute solutions. In his own words [6]:

'I wish to make a remark on this matter which appears to me to render intuitive an important proposition which the kinetic theory of fluids established in a somewhat laborious manner. Van't Hoff law tells us that a molecule of ethyl alcohol in solution in water has the same energy as one of the molecules of the vapor over the solution; it would still have this energy if it were present in chloroform (that is to say if it were surrounded by chloroform molecules), or even if it were in methyl or propyl alcohol; this indifference to the nature of the molecules in which it moves makes it almost impossible to believe that it would not still have the same energy if it were in ethyl alcohol... we can now say: At the same temperature all the molecules of all fluids have the same mean kinetic energy, which is proportional to the absolute temperature.'

This elegant argument, still to me the most intuitive explanation of why the kinetic energy of molecules in a liquid is the same as in the gaseous state, led Perrin to assume that, arguably, the mean kinetic energy is the same also for a 'dust' made of many molecules, and allowed him to account for his data independently from the general approach by Einstein and Smoluchowski. Perrin also had the good habit, basically forgotten in present-day papers, of reporting on experimental problems and dead-ends, and, better still, of inquiring into their origin. By questioning about the larger apparent density of natural gamboge emulsions compared to the value found for samples carefully purified in alcohols, he was led to conclude that the former contained 'a colloid with invisible granules', which could 'coat the large granules of resins and change their apparent density'. Cleverly, Perrin was able to turn this nuisance into a useful tool:

'... I am content to say that very minute quantity of a protecting colloid, precisely such as it is present in the natural latex of gamboge, added to the emulsion studied, prevent the granules from caking together in water acidulated by pure hydrochloric acid.'

Hence, he fully recognized that small colloidal particles can stabilize emulsion droplets by adsorbing at the interface, and even managed to exploit this effect more or less at the time when Pickering, after a preliminary observation by Ramsden [7], first described these systems in detail [8].

An exponentially decreasing profile of the particle concentration with the height from the cell bottom is expected only provided that the suspension behaves as an ideal gas, namely, that interparticle interactions can be fully neglected. Perrin was, however, well aware that colloidal science could be developed well beyond the single particle limit. Indeed, while recalling that he could apply van 't Hoff law only because of the negligible concentration of the suspensions he used, compared to the systems investigated by standard osmotic pressure measurements, he argues that a generalization 'more or less analogous to that of van der Waals' could allow us to evaluate, one day, the osmotic pressure of these solutions: another brilliant anticipation. What Perrin could not foretell is how much the statistical mechanics of colloidal fluids would profit from his seminal sedimentation studies.

Some of these interesting advancements are discussed in this review. In the next section, we shall first see that equilibrium settling profiles yield crucial information on the phase behavior of model colloidal suspensions of hard and 'sticky' spheres, whereas for charged spheres sedimentation has in store an instructive surprise. This survey is then extended to more complex systems, ranging from suspensions of anisotropic particles, to soft colloidal solids, dispersions of 'active' Brownian particles, self-aggregating surfactant and biological solutions. Section 3 is conversely dedicated to settling dynamics, a much more challenging subject where sedimentation, like the Roman god Janus with both a warlike and a peaceful face, unravels its double-faced nature of a macroscopically slow and regular process stemming from a microscopic chaotic world. A final surprise is discussed in section 4, where even one of the oldest laws of physics, the Archimedes' Principle, is challenged, and shown to require substantial corrections when applied to particle sedimentation in a correlated fluid.

\section{Sedimentation equilibrium}

\subsection{Gravitational length and the colloidal regime}

The basic result of Perrin's investigation was proving that, at equilibrium in the presence of gravity, a dilute suspension of equal-sized ('monodisperse') particles of mass $m$ takes on an inhomogeneous density profile along the vertical direction $z$ that mirrors the barometric law for ideal gases, $n(z)=n(0)$ $\exp \left(-z / \ell_{\mathrm{g}}\right)$, where $n$ is the number of particles per unit volume and $\ell_{\mathrm{g}}$, called the gravitational (or sedimentation) length, is given by the ratio of the thermal energy $k_{\mathrm{B}} T$ to the magnitude of total force acting on the particle, which is the sum of the particle weight $-m g \hat{\boldsymbol{z}}$ with the buoyant force $\boldsymbol{F}_{b}$ provided by the surrounding fluid. For the moment, we shall assume that the latter is simply given, according to the Archimedes' principle, by the weight of a volume of fluid equal to the particle volume $V_{\mathrm{p}}$. Thus, by introducing the buoyant mass $^{2} \quad m^{*}=\Delta \rho V_{\mathrm{p}}$,

${ }^{2}$ In a rigorous thermodynamic analysis, the buoyant mass should rather be written as $m^{*}=\left(1-\rho_{0} \bar{v}\right) m$, where the specific volume $\bar{v}$ of the solute can depend on its interactions with the solvent and on the thermodynamic conditions of the latter: the microscopic meaning of $\bar{v}$ will be discussed in section 4. Actually, $\boldsymbol{F}_{b}$ stems from the pressure gradient $\mathrm{d} P / \mathrm{d} z=-\rho_{0} g$ that builds up almost instantaneously only if the solvent is incompressible. In section 4 we shall also see that, when the host fluid is itself a suspension of different particles (hence compressible), the effective buoyancy changes with time and position in the cell while the host fluid settles to equilibrium. 
where $\Delta \rho$ is the difference between the particle and solvent densities $\rho_{p}$ and $\rho_{0}$, we have

$$
\ell_{\mathrm{g}}=\frac{k_{B} T}{\Delta \rho V_{p} g}=\left(\beta m^{*} g\right)^{-1},
$$

where $\beta=1 / k_{\mathrm{B}} T$. The dimensionless ratio $a / l_{\mathrm{g}}$, where for a dilute suspension $a$ is the characteristic size of the particles (their radius, for spheres) or, more appropriately, the density correlation range set by interparticle forces in the general case, allows us to set apart two radically different experimental situations, traditionally called the 'Brownian' $\left(a / l_{\mathrm{g}} \ll 1\right)$ and 'non-Brownian' $\left(a / l_{\mathrm{g}} \gg 1\right)$ regimes. Consider first the equilibrium state that sets in when the particle weight eventually gets balanced by the pressure gradient that builds up due to particle accumulation at the bottom of the container: this state is characterized by a particle concentration profile $n(z)$ that, as we shall shortly see, is fully ruled by $\ell_{\mathrm{g}}$. When $a / l_{\mathrm{g}} \ll 1, n(z)$ varies appreciably only over distances that are much larger than any microscopic length scales, whether the particle size or, more generally, the correlation range. Conversely, when $a / l_{\mathrm{g}} \gg 1$ the profile displays strong oscillations on the particle scale ('layering') and, in general, the local thermodynamic quantities do not depend only on $n(z)$, but on its spatial derivatives too. The macroscopic equilibrium equations we shall write assume $a / l_{\mathrm{g}} \ll 1$ as a basic condition, namely, they entail a 'local density approximation' ${ }^{3}$.

The ratio $a / l_{\mathrm{g}}$ is even more significant for the dynamics of the sedimentation process. We can indeed dub a particle 'Brownian' when, on the microscopic length scale, the contribution of gravity settling to its motion is just a small perturbation with respect to thermal agitation. Quantitatively, this means that $t_{b} / t_{s} \ll 1$, where $t_{b}$ and $t_{s}$ are the times it takes for the particle to diffuse and, respectively, to settle, over its own size (the latter is usually known as the 'Stokes time'). Since $t_{b} \sim a^{2} / D_{0}$, where $D_{0}$ is the Brownian single-particle (self) diffusion coefficient, and $t_{s} \sim a / v_{\mathrm{s}}$, where $v_{\mathrm{s}}$ is the steady-state settling velocity for an isolated particle, we have

$$
\frac{t_{b}}{t_{s}}=\frac{a v_{s}}{D_{0}}=P e,
$$

which is the Péclet number, playing in hydrodynamics the role of the ratio between advective and diffusive transport. Because of Einstein's relation $D_{0}=k_{\mathrm{B}} T / f$, where $f$ is the viscous friction coefficient, and since at steady-state $v_{0}=$ $m^{*} g / f$, we have once more $P e=a / \ell_{\mathrm{g}}$. Again, in the presence of direct interparticle forces generating structural correlations in the suspension, $a$ should rather be taken as the correlation range of the latter. We shall indeed see that the effect of particle motion on the surrounding solvent induces 'hydrodynamic' interactions which, when $P e \gg 1$, substantially perturb the equilibrium structure of the suspension. As a matter of fact, the occurrence of a nonequilibrium structure factor is arguably the source of several puzzling effects in non-Brownian particle settling that are discussed in section 3.3.

3 Some experiments discussed in section 2.5 [9], however, suggest that a local density approximation may still give reasonable results even when $a / \ell_{\mathrm{g}} \gtrsim$ $1-2$, yielding profiles which are just a coarse-grained version on the particle scale of the true ones.
Hence, for several reasons, the settling of non-Brownian particles is still a very open subject, often involving extremely challenging questions in fluid dynamics and nonequilibrium statistical mechanics [10]. Because of this, this review is mostly concerned with particle settling in the Brownian regime, although we shall occasionally venture into the high $P e$ numbers regime. It is useful to point out that, for spherical particles, the condition $P e=1$ corresponds at room temperature to

$$
a=\left(\frac{3 k_{\mathrm{B}} T}{4 \pi \Delta \rho g}\right)^{1 / 4} \simeq\left(\frac{0.1}{\Delta \rho}\right)^{1 / 4} \times 10^{-4} \mathrm{~cm} .
$$

Hence, because of the strong dependence of $P e$ on size $\left(\mathrm{Pe} \propto a^{4}\right)$, the transition between the two regimes takes place for $a$ in the quite narrow range $0.5 \mu \mathrm{m}<a<1.5 \mu \mathrm{m}$ even if $\Delta \rho$ is varied between 0.02 and $2 \mathrm{~g} \mathrm{~cm}^{-3}$ (a range covering most common colloids). ${ }^{4}$ Of course the criterion $P e=1$ is just a practical convention, and this transition may be rather smooth: hence the specific dependence on $P e$ is actually expected to depend on the investigated structural or dynamic effecAlthough we have discussed only natural settling, similar considerations apply to forced sedimentation too, provided the $g$ stands for the centripetal acceleration $\omega^{2} r$, where $\omega$ is the angular frequency of rotation of the centrifuge. Of course, one should carefully take into account that, in this case, the force acting on the particle grows linearly with the distance $r$ from the axis of rotation, so that the 'barometric' profile for a dilute suspension is in this case an exponential in $r^{2}$. We also recall that in centrifugation studies the particle settling velocity is usually rescaled to the acceleration by defining a 'sedimentation coefficient' $s=v_{0} / \omega^{2} r$, which has then the dimensions of a time and is usually measured in svedbergs ( $\mathrm{S})$, where $1 \mathrm{~S}=0.1 \mathrm{ps}$, a particularly convenient unit for the rotation speed achievable with ultracentrifuges (for $\omega^{2} r=10^{6} \mathrm{~g}$, a macromolecule with $s=1 \mathrm{~S}$ settles at $v_{s}=1 \mu \mathrm{m} \mathrm{s}^{-1}$ ). By using Einstein's relation again, one easily gets the Svedberg equation $s=\beta m^{*} D_{0}$, which allows the comparison of sedimentation data to values of $D_{0}$ obtained for instance by dynamic light scattering ${ }^{5}$.

\subsection{Thermodynamics in a single shot: equations of state and phase diagrams}

Colloids have proved to be a valuable test bench for basic ideas in condensed matter physics. Basically, their power as model systems results from the possibility of tracing out the degrees of freedom of the solvent and of any other small molecular components, effectively 'mapping' a multicomponent suspension onto a system of particles interacting via an effective potential $u(\{\boldsymbol{r}\})$, which explicitly depends only on the positions $\{\boldsymbol{r}\}$ of the colloidal particles, but can actually

4 The gamboge particles used by Perrin had $a=0.212 \mu \mathrm{m}, \Delta \rho=$ $0.21 \mathrm{~g} \mathrm{~cm}^{-3}$, so that $\ell_{\mathrm{g}} \simeq 50 \mu \mathrm{m}, P e \simeq 0.04$. The corresponding data for the mastic emulsions are $a=0.52 \mu \mathrm{m}, \Delta \rho=0.067 \mathrm{~g} \mathrm{~cm}^{-3}, \ell_{\mathrm{g}} \simeq 10 \mu \mathrm{m}$, $P e \simeq 0.05$.

5 It is also useful to point out that clever methods have been devised to slow down sedimentation, so to simulate (time-averaged) microgravity conditions [11]. These techniques can profitably been used to investigate suspensions of particles at low effective $P e$ numbers. 
be tuned by controlling the composition of the solvent [12]. This is also called a 'potential of the mean force', because $-\nabla_{r_{i}} u(\{\boldsymbol{r}\})$ gives the force on a particle $i$ averaged over the configurations $\left\{\boldsymbol{r}^{\prime}\right\}$ of the solvent molecules for a fixed configuration $\left\{\boldsymbol{r}_{j \neq i}\right\}$ of the other colloidal particles [13]. In this scheme, the role of the pressure for a single-component fluid is taken on by the osmotic pressure $\Pi$ : for instance, the law of ideal gases $P=n k_{\mathrm{B}} T$ becomes, for non-interacting colloidal particles, the van 't Hoff law $\Pi=n k_{\mathrm{B}} T$ used by Perrin to account for his findings.

For the purposes of statistical mechanics, a very interesting feature of colloid sedimentation is that a single measurement of the equilibrium profile induced by gravity in a suspension allows us, in principle, to reconstruct the entire equation of state (EOS) of the system. The macroscopic settling process of a single-component colloidal system comes to rest when the pressure distribution in the suspension satisfies $\nabla p=-\rho \boldsymbol{g}$, where $\rho$ is the density of the suspension, which can be written in terms of the local particle volume (or packing) fraction $\phi=V_{p} n$ as $\rho=\rho_{0}+\Delta \rho \phi$. This hydrostatic equilibrium condition easily yields an equation for the profile along the (upward directed) vertical axis $z$ of the osmotic pressure of the colloids ${ }^{6}$

$$
\frac{\mathrm{d} \Pi(z)}{\mathrm{d} z}=-\Delta \rho g \phi(z) .
$$

If the suspension, containing a total number of particles $N$, is placed in a rectangular vessel of horizontal cross-section $S$ and height $h$, a straightforward integration of equation (5) $\mathrm{fixes}$ the pressure at the bottom of the container to $\Pi(0)$ $=m g n_{s}$, where $n_{s}=N / S=n h$ is the number of particles per unit surface. By introducing the (osmotic) isothermal compressibility $\chi_{T}=k_{\mathrm{B}} T(\partial \Pi / \partial n)^{-1}=\left(k_{\mathrm{B}} T / V_{\mathrm{p}}\right)(\partial \Pi / \partial \phi)$ -1 , we have

$$
\frac{\mathrm{d} \phi(z)}{\mathrm{d} z}=-\frac{\chi_{T}(\phi)}{\ell_{\mathrm{g}}} \phi(z),
$$

which allows us to obtain the sedimentation profile once the EOS of the system $\Pi=\Pi(\phi)$ is known. Since experiments yield $\phi(z)$, it is more useful to proceed the other way around: by integrating equation (5) up to the top $z=h$ of the sample, usually devoid of particles at the end of the settling process $(\Pi(h) \simeq 0)$, we get

$$
\Pi(z)=\Delta \rho g \int_{z}^{h} \phi(z) \mathrm{d} z,
$$

which simply means that at equilibrium the osmotic pressure $\Pi(z)$ at a given height $z$ has to match the weight per unit surface of the particles lying above that level. Hence, by pairing the experimental data for $\phi(z)$ and $\Pi(z)$, one gets the osmotic $\operatorname{EOS} \Pi(\phi)$ of the colloid up to the maximal volume fraction $\phi(0)$ attained by the suspension at the cell bottom ${ }^{7}$.

\footnotetext{
${ }^{6}$ Equation (5) can be rigorously justified by density functional theory (DFT) only provided that that the free energy density is a local functional $\mathcal{F}[n(z)]$ of $n(z)$, which, as we mentioned, requires $\ell_{\mathrm{g}}$ to be much larger than any microscopic length scales [14].

${ }^{7}$ Note that by driving and confining the particles to the bottom, gravity, in effect, acts as a kind of osmotic membrane (osmotic pressure always arises from confinement, see for instance [15], chapter 7).
}

Thus, measurements of the equilibrium sedimentation profile provide the most important piece of information about the thermodynamics of a colloidal system; this has permitted us to perform accurate tests of model systems in statistical mechanics, which we shall discuss after some preliminary experimental considerations.

\subsection{The hard life of Perrin's disciples}

Measuring the concentration profile up to high particle fractions is not trivial. For large sample batches, a simple approach consists of sampling the equilibrated suspensions at different heights, and obtaining $\phi(z)$ via direct density measurements. Although successfully used in early studies to investigate the freezing of a suspension into an ordered colloidal crystal [16], this method lacks, however, the resolution required for determining the full EOS. As today we have fast cameras and confocal microscopes at our disposal, which effectively reject stray light, one may also think of extending Perrin's approach to much more concentrated suspensions. Yet, because of the limited working distance of high numerical aperture objectives, video microscopy studies have in the past mostly been used to investigate thin samples. Optical schemes based on visualizing the local concentration of the particles with a microscope whose optical axis is placed along the horizontal (which can be obtained by simply tilting by $90^{\circ}$ a commercial confocal instrument, or by developing a custom optical setup) and by scanning the sample along the vertical direction, have however been developed (see, for instance, [17]), and recently applied to sedimentation [18]. Although probably not yet as accurate as the scattering methods described in what follows, these imaging techniques present the noticeable advantage of providing a real-space visualization of the particle distribution, which can be extremely useful in investigating the structure of the ordered or glassy solid phases that may form in the denser region of the profile. Note, however, that a detailed spatial analysis requires the particles to be optically resolvable; hence, because of equation (4), these methods are more suitable for investigating suspensions close to or within the non-Brownian regime.

Since colloids that are optically transparent up to high concentration can currently be prepared by using particles matched in refractive index with the solvent, light scattering seems to be another natural choice. Yet, the intensity of the light scattered by a suspension depends not only on the local concentration, but on interparticle interactions too, and de-convolving structure factor effects is not easy ${ }^{8}$. A way

${ }^{8}$ Instead of scattering, one may however think of exploiting absorption, which is of course linear in $\phi(z)$, as made for instance by Rutgers et al [19] by using space-resolved $\mathrm{x}$-ray densitometry. Besides requiring complex instrumentation and careful de-convolution of the rough data, this method is suited for particles made of a material with an electron density close to that of the solvent, to avoid having an excessively large absorption cross-section (for instance, polystyrene particles in water); since this usually means that also the material densities of particle and solvent are closely matched, reaching sedimentation equilibrium may require, for these suspensions, a very long time. Optical absorption can however be exploited for special particles, such as the ferrofluids we discuss in 2.6: using micro-capillary cells, this can allow to obtain quantitative concentration profiles up to rather high $\phi[20]$. 
to circumvent this problem is by using particles that are optically anisotropic: similarly to what occurs in incoherent neutron scattering, the light scattered from particles of this kind contains a depolarized component whose intensity, provided that interparticle forces have spherical symmetry, is strictly proportional to $\phi(z)$ [21]. Depolarized light scattering (DeLS) is then an accurate probe of the sedimentation profiles with a dynamic range of several decades in volume fraction, and the crucial advantage that absolute calibration in $\phi$ is simply obtained by comparison with a reference sample. As a matter of fact, most of the experiments performed by my group have exploited the unique material and optical properties of colloidal particles made of MFA (methyl fluoroalkoxy resin), a copolymer of terafluoroethylene and perfluoromethyl vinyl ether, which, although spherical, have a partially crystalline interior and are therefore birefringent.

Particles with these properties, however, are neither common nor easy to synthesize. An alternative simpler optical technique that does not require particles with special optical properties and yields sedimentation profiles which can be even superior in terms of noise to those obtained by DeLS is beam deflection (BD, [22]), which exploits the deflection of a laser beam propagating through a medium where a concentration, and therefore refractive index gradient, is induced by an external field-a kind of 'mirage' effect. The BD signal is proportional to $\mathrm{d} n / \mathrm{d} z$ and therefore to the derivative of the concentration profile; the raw data must then be integrated twice to obtain the EOS. While this is not a problem for smooth profiles (on the contrary, the additional integration helps smoothing noisy data), it is clearly a drawback when dealing with the discontinuous refractive index occurring when two distinct phases coexist in the equilibrium profile. From equation (6) it is also easy to show that the $\mathrm{BD}$ signal is actually proportional to the polarized light scattering intensity at zero scattering angle.

\subsection{When entropy rules: hard and sticky spheres}

One of the most important achievements of soft matter science has been showing that colloids can give full support to the Kirkwood-Alder freezing transition; namely, to the fact that, beyond a packing fraction of about $50 \%$, a system of hard spheres (HS) undergoes a spontaneous entropy-driven transition to a crystal state [23, 24]. In 1993, measurements of the equilibrium sedimentation profile [25] showed that the whole fluid phase of a suitably prepared colloidal suspension is very well fitted by the Carnahan-Starling (CS) EOS for HS [26]

$$
Z_{f}(\phi)=\frac{1+\phi+\phi^{2}-\phi^{3}}{(1-\phi)^{3}}
$$

where the pressure is rescaled to the ideal gas value by introducing the compressibility factor $Z(\phi)=\beta \Pi(\phi) / n$. A short time later, Rutgers et al [19] proved that also the colloidal crystal phase conforms quite well to the approximate EOS for a HS solid obtained by a van der Waals mean field approximation [27]

$$
Z_{c}(\phi)=\frac{3}{1-\phi / \phi_{\text {ocp }}}
$$

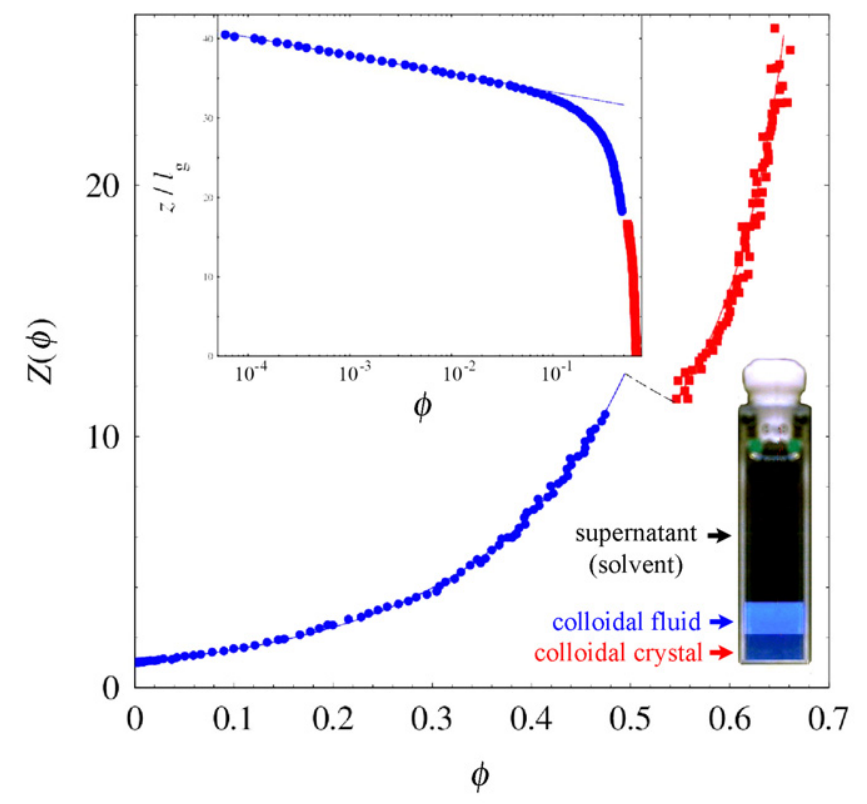

Figure 1. Inset: equilibrium sedimentation profile, scaled to the gravitational length $\ell_{\mathrm{g}}=210 \pm 5 \mu \mathrm{m}$, for aqueous suspensions of MFA particles with radius $a=82 \pm 3 \mathrm{~nm}$, in the presence of $100 \mathrm{mM} \mathrm{NaCl}$ added to screen electrostatic interactions. The line shows that the barometric region extends only up to a volume fraction $\phi \simeq 0.03$. Body: EOS (compressibility factor $\left.Z(\phi)=\Pi(\phi) / n k_{\mathrm{B}} T\right)$ obtained from equation (7), with the fluid branch shown by dots and the HS colloidal crystal by squares. The full lines are, respectively, the CS EOS for the fluid (8) and the van der Waals approximation for the solid (9) (data from [28]).

where $\phi_{\text {ocp }}=\pi /(3 \sqrt{2}) \simeq 0.74$ is the ordered close-packing fraction for HS. Figure 1, where more recent data are presented, shows how close a real colloidal system can be to the idealized HS model. Note in particular that both the fluid and the solid EOS are directly plotted over the data with no fit parameter.

If the spontaneous freezing of HS is anything but obvious, more surprises come from considering interaction potentials with an additional attractive tail having a short range $\delta$ outside the particle hard-core diameter $\sigma$. Numerical simulations by Hagen and Frenkel [29] have indeed shown that, when

$\delta / \sigma \lesssim 1 / 3$, crystallization precedes gas condensation, the liquid phase in fact disappears, and the system just displays a single stable fluid phase. In addition, when the attractive contribution is very short-ranged $(\delta / \sigma \lesssim 0.06)$, two distinct stable solid phases appear, with the same face-centered cubic structure (like a HS solid), but a different density [30] ${ }^{9}$. This scenario, which is not observed for simple fluids (where usually $\delta>\sigma$ ), is far from being alien to soft matter systems; on the contrary, a large variety of engaging phenomena, ranging from crystallization in protein solutions [31] to clustering and gelation in colloidal suspensions [32], stems from the occurrence of very short-ranged attractive forces.

A paradigmatic example of short-range attractions are the depletion interactions induced in a colloidal suspension by

${ }^{9}$ This first-order isostructural transition is actually reminiscent of the liquidvapor transition in the van der Waals description of fluids with longer-ranged attractive forces; in fact, because no symmetry-breaking takes place, the coexistence curve of the two solid phases ends in a critical point. 
the addition of high molecular weight additives such as nonadsorbing polymers or surfactant micelles (hence themselves smaller colloidal particles) [33]. Although they can be given an intuitive interpretation as an osmotic effect, depletion forces actually have a purely entropic origin. A large particle acts indeed as an impenetrable obstacle that excludes the center of a small particle of size $\delta$ from a sphere of diameter $\sigma$ $+\delta$ : pushing two large particles close together to the point that their depletion regions overlap, reduces this excluded volume, so that the region of motion, (and therefore the entropy) of the small particles increases. The resulting effective attractive interactions between the large particles, which may eventually separate out into a condensed phase, result then from the 'struggle' of the small particles to maximize their entropy. The phase diagram of depletion system strongly depends on the size ratio $q=\delta / \sigma$ [33]. For very short-ranged depletion it is particularly useful considering the limit of a vanishing range of the attractive term, obtained by shrinking the width of a square-well (SW) potential and concurrently increasing its depth, so that the thermodynamic quantities remain finite, ${ }^{10}$ which can be formally done py taking the limit $\delta \rightarrow 0$ of

$$
\beta u(r)= \begin{cases}\infty & 0 \leqslant r \leqslant \sigma \\ \ln [12 \tau \delta /(\sigma+\delta)] & \sigma<r \leqslant \sigma+\delta \\ 0 & r>\sigma+\delta .\end{cases}
$$

This limiting interaction, fixed by a single 'stickiness parameter' $\tau$ inversely proportional to the strength of the attractive forces, defines the adhesive (or 'sticky') HS (AHS) model [35].

Since depletion forces are also believed to be at the origin of many effects in biology related to the so-called 'macromolecular crowding' [36], it is not surprising that strong efforts have been made to investigate the phase behavior of model depletion systems. However, a detailed comparison to the theoretical predictions requires us to relate the concentration of the macromolecular additive to the parameters of the potential. This can accurately be done by obtaining the EOS from equilibrium sedimentation measurements. By adding an increasing amount of a nonionic surfactant (Triton X100), which forms globular micelles with a radius of about $3.5 \mathrm{~nm}$, depletion interactions can be progressively switched on among the MFA particles whose EOS in the absence of surfactant is shown in figure 1 . The very small size ratio $q \simeq 0.03$ then allows us to fit the EOS obtained from the equilibrium sedimentation profiles by a reliable analytical expression for the EOS for AHS [37],

with fitted values for $\tau$ covering the range $0.2 \lesssim \tau \lesssim 2$, and for an accurate assessment of the strength of the depletion potential versus the experimental depletant concentration [28]. The experimental phase diagram, compared in figure 2 to the theoretical predictions for a SW potential with $q=0.03$ [38]

${ }^{10}$ For instance, the limiting potential obtained from equation (10) yields a second virial coefficient for the pressure given by $B_{2}(\tau)=B_{2}^{\mathrm{hs}}(1-1 / 4 \tau)$, where $B_{2}^{\text {hs }}$ is the value for hard spheres. It is however worth pointing out that, although in practice the EOS of a system of particles interacting via an attractive potential with very short but finite range closely resembles the AHS model, the formal limit of the potential in (10) for strictly monodisperse spheres display some thermodynamic inconsistencies [34].

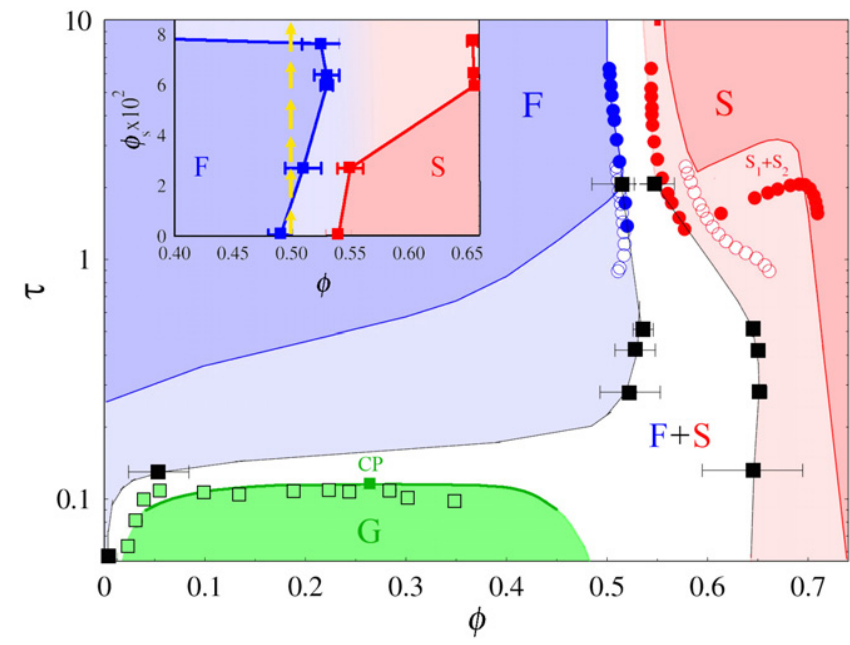

Figure 2. Comparison of the experimental phase boundaries (squares, [28]) for colloidal particles interacting via very short-ranged depletion forces induced by surfactant micelles, with the theoretical phase diagram for a square-well (SW) potential with $q=0.03$ [38] (full lines) and to numerical simulations for the same potential, but with finite particle size polydispersity (open and closed dots) [39]. The open squares are the experimental L-L phaseseparation points, which are compared to the AHS fluid-fluid coexistence curve [37]. The re-entrant melting behavior is better appreciated in the inset, where the phase boundaries are plotted versus the depletant concentration.

(mapped on a $\tau$-axis by virial coefficient matching), reserves some surprises, for the fluid-solid coexistence region is much narrower than theoretically predicted, and no evidence of an isostructural solid-solid phase separation is found. A plausible explanation of these discrepancies is however even more surprising: numerical simulations by Bolhuis and Frenkel [39], also displayed in figure 2, show indeed that a dispersion in the particle size as small as $1 \%$ totally wipes out the isostructural transition and noticeably shrinks the coexistence gap. Hence, these results say that a suspension of particles that in the vast majority of the experiments on colloid science would be regarded as very monodisperse may show a phase behavior which widely deviates from the ideal. Another delicate feature of the experimental phase diagram, better appreciated in the inset of figure 2 , is that, close to the HS limit, the freezing line shows a positive slope, namely, the limiting fluid density first increases with the added surfactant concentration, to then plummet rapidly to very low $\phi$ values. This means that a suspension prepared at $\phi \simeq 0.50-0.53$ (within the HS fluid/solid coexistence region) first fully melts, to recrystallize only for consistently stronger attractive forces. Such a peculiar 're-entrant melting' scenario for shortranged attractive potential, quite uncommon in condensed matter, has actually been theoretically predicted [38].

But the most interesting evidence obtained by sedimentation studies of systems with short-ranged attractions concerns the formation of disordered solids. When the concentration of depletant is large, the freezing of a dilute suspension into a colloidal crystal is usually quenched, with the whole system rather turning into a tenuous but mechanically solid structure with no crystalline order, a 
colloidal gel. Albeit metastable, the liquid-vapor coexistence line, which for a suspension actually marks a liquid-liquid (LL) phase separation, still affects the system phase behavior; for instance, the crystal nucleation rate strongly increases close to metastable L-L critical point [40]. In [28] gel formation was found to be tightly related to $\mathrm{L}-\mathrm{L}$ phases separation; indeed, arrested gel phases seem to form only when the system is quenched inside the metastable coexistence region. Thus, it is the phase-separation process that drives the formation of depletion gels (which, however, may be so weak as to break, because of gravity stresses, into clusters that settle very fast and recompact into a denser disordered structure). The general validity of this 'spinodal decomposition' route to gelation has later been confirmed by simulations and confocal microscopy studies of other colloidal systems [41].

Finally, it is important to point out that the derivation of the EOS and phase diagram from the experiments in [28] relies on two distinctive features of the investigated depletion system, namely, that (i) the gravitation length of the depletant is so large that no appreciable settling of the latter takes place, and (ii) $q$ is so small that changes along $z$ of the depletant volume fraction, calculated with respect to the solvent volume $(1-\phi) V$, is negligible, which ensures that depletant 'partitioning' effects are marginal [42]. If any of these two conditions is not met, the profile integration does not yield the EOS for a fixed interparticle potential, because the strength of the latter varies along the cell. In the presence of depletant sedimentation, moreover, the profile can display very curious effects, such as a dependence on the total height $h$ of the initially homogeneous

suspension. For instance, when $q \gtrsim 0.4$, the phase diagram of a depleted colloid displays a stable $\mathrm{L}-\mathrm{L}$ phase-separation region; yet, two distinct phases can be seen in the equilibrium profile only when the ratio $h / \ell_{\mathrm{g}}$ is smaller than a critical value [43].

\subsection{When entropy conspires with electrostatics: sedimentation of charged colloids}

Colloidal particles often get charged by being released into the solvent small ions (counterions) of the opposite sign, so they interact via screened electrostatic forces that are often welldescribed by a Yukawa repulsive potential. While settling, a charged particle brings along the surrounding counterion cloud, the so-called 'double layer', which, when integrated over distances much larger than the Debye-Hückel screening length, fully screens its charge. Yet, counterions are definitely not willing to settle; because of their huge $\ell_{\mathrm{g}}$, they very much prefer wandering through the whole volume. May this tug-ofwar between the requirements of charge neutrality and maximization of the entropy of counterions result in a slight shift of the density profile of the latter with respect to the particle distribution? In other words, could a suspension of charged particles at sedimentation equilibrium display an internal polarization field, partially balancing gravity?This eventuality, originally suggested in [25] to account for some discrepancies in the experimental value of $\ell_{\mathrm{g}}$, was investigated by Biben and Hansen, who found that a suspension of fully de-ionized colloidal particles may indeed behave as a kind of 'colloidal capacitor' [44]. Some years later Philipse and Koenderink [45] actually reported the formation of strongly 'inflated' equilibrium sedimentation profiles for charged silica spheres in ethanol and attributed them to a Donnan equilibrium [46] that, for charged colloids, implies for instance that the partitioning of the small ions (counterions and co-ions) across a semipermeable membrane generates an electric field across the latter. For a suspension of particles bearing $Z$ electron charges, the Donnan effect modifies the EOS by introducing an additional term [47]

$$
\beta \Pi(y)=\beta \Pi(0)+2 n_{s}\left[\sqrt{1+y^{2}}-1\right],
$$

where $\Pi(0)$ is the EOS for $Z=0$, and $y=Z n /\left(2 n_{s}\right)$ is the ratio between the number density of the particle counterions $Z n$ and the total number density $2 n_{s}$ of cations and anions in the reservoir (due to the addition of salts or already present in the solvent), which is related to the Donnan electrostatic potential $\psi$ by $y=-\sinh (\beta e \psi)$. The EOS can therefore consistently differ from the van 't Hoff law $\beta \Pi(0)=n$ even for a very dilute suspension. In the context of sedimentation, the Donnan term, which is solely due to the entropy gain of the counterions and quantitatively accounts for the physical mechanisms suggested in [25], clearly depends on the local particle number density $n(z)$ along the profile ${ }^{11}$. When equation (11) is used to evaluate the equilibrium profile, two limiting regimes can be clearly spotted: whereas at high salt concentration $(r \ll 1$, which is the case for the particles used in [25]) one recovers the standard barometric decay, for vanishing ionic strength, $r \rightarrow \infty$, the decay is still exponential, but with a decay

length $\ell_{\mathrm{g}}^{*}=(1+Z) \ell_{\mathrm{g}}$ that, for the usual values of colloidal particle charge, can be three orders of magnitude larger than $\ell_{\mathrm{g}}$. These ideas were given rigorous theoretical bases by van Roij [48] who, starting from a hydrostatic equilibrium condition obtained by considering explicitly both the colloidal particles and the small ions, showed that, in the absence of interparticle interactions, three regions can be distinguished in the profile. The standard barometric region is recovered only when $n(z)<n_{1}=2 n_{s} / Z^{2}$ (which is a vanishing small region when $n_{s} \rightarrow 0$ ), while the hugely inflated exponential

zone with a decay length $\ell_{\mathrm{g}}^{*}$ conversely occurs when $n(z)>$ $n_{2}=2 n_{s} / Z$. In between, a linear decay of the concentration profile with slope $\left(Z \ell_{\mathrm{g}}\right)^{-1}$ is predicted. Since $n_{2} / n_{1}=Z$, this region typically spans 2-3 orders of magnitude in $\phi$, accounting therefore for most of the sedimentation profile of highly charged colloids. As a matter of fact, Raşa et al later managed to observe these regimes and to detect the predicted electric field [49, 50].

There was however something disquieting, dare I say almost 'heretical', in this approach to the problem, for it questioned one of the basic pillars on which colloid physics stands: namely, as we already mentioned, that a hotchpotch of particles, solvent, small ions, and usually other additives can be conceptually but rigorously reduced to a much simpler one-component system of particles interacting via an effective potential. For some reason, expressly

\footnotetext{
${ }^{11}$ Recalling that gravity effectively acts as a 'generalized osmotic membrane', the similarity between sedimentation and membrane equilibrium is evident.
} 
considering the small ions seemed to be necessary; yet, as pointed out by Belloni [51], the entropy of the counterions and the electric field generated by the spatial inhomogeneity should be already, albeit implicitly, taken into account in the structure factor of the effective one-component system through the compressibility equation and the local electroneutrality condition. The equivalence of these two viewpoints was indeed verified by Torres et al [52] by comparing for real experimental conditions [9] the so-called primitive model, where a threecomponent system of colloids, cations, and anions with unscreened Coulomb interactions is considered, to a simpler one-component model of particles interacting through pairwise screened-Coulomb repulsions. Nevertheless, the issue of charged colloid sedimentation still held surprises, for the same group later showed that, in the limit of vanishing ionic strength, the one-component model based on a Yukawa pair potential breaks down: recovering consistence with the primitive model actually requires considering many-body effects, which cannot be embodied in a pairwise interaction [53]. A brilliant way to bypass the hard task of dealing with many-body contributions is introducing a so-called 'volume term'. We have indeed omitted a subtlety in the formal scheme that maps a colloidal suspension onto an effective one-component system: besides the potential of the mean force, which contains pair or higherorder interparticle interactions, the effective Hamiltonian also contains a one-body 'volume term', which does not depend on the particle coordinates, but does depend on the particle concentration and on the thermodynamic state of the 'solvent' (which, we stress again, includes all the molecular species, then also the small ions) [13]. If this term is included in the effective one-component description, the agreement with the primitive model is very good. Equilibrium sedimentation experiments on highly de-ionized colloidal suspensions allow us to highlight the important role played by one-body volume terms that have often been overlooked in the past, and actually account for several other puzzling effects taking place when the solvent is spatially inhomogeneous.

\subsection{Novel forces, novel shapes, novel challenges}

Hard, sticky, and charged spheres, although simple and powerful model systems, do not exhaust the catalogue of interesting and practically valuable colloidal fluids. Widening the scene by considering other forms of the interaction potential, or even more by studying particles with an anisotropic shape, brings in new challenging questions. Ferrofluids made of nanoparticles behaving as single magnetic domains, and therefore interacting via tunable dipole forces, are of current interest not only for applications, but also for basic condensed matter theory. By means of a dedicated centrifuge, particularly useful for investigating the equilibrium sedimentation profiles of small colloidal particles, Luigjes et al $[20,54]$ have investigated suspensions of two kinds of monodisperse magnetite $\left(\mathrm{Fe}_{3} \mathrm{O}_{4}\right)$ nanoparticles, with core diameters of $11 \mathrm{~nm}$ and $13.4 \mathrm{~nm}$ respectively. Since magnetic particles strongly absorb light, measurements were limited to $\phi \lesssim 10-15 \%$, which, although too low to extract the full EOS, is still large enough to obtain with confidence the lowest order deviation from the van 't Hoff law (the second osmotic virial coefficient $B_{2}$ ). For sufficiently dilute suspensions, the effective interaction between the magnetic particles can be simply obtained by averaging the magnetic dipole interaction $u(\boldsymbol{r}, \Omega)$, where $\Omega$ denotes the angular orientation of the dipoles, according to the standard procedure for obtaining a potential of the mean force: $\lim _{n \rightarrow 0} \exp [-\beta u(r)]=$ $\langle\exp [-\beta u(\boldsymbol{r}, \Omega)]\rangle_{\Omega}$. This potential is determined by a single parameter $\lambda=\beta \mu_{0} \mu^{2} /\left(4 \pi \sigma^{3}\right)$, where $\sigma$ and $\mu$ are the particle diameter and magnetic moment, and $\mu_{0}$ the vacuum permeability. For small coupling, $\lambda \lesssim 2.5$, one finds:

$$
\frac{B_{2}}{B_{2}^{\mathrm{hs}}}=1-\frac{\lambda^{2}}{3}-\frac{\lambda^{4}}{75}+c \lambda^{6},
$$

with $c \simeq 5 \times 10^{-4}$, which corresponds to a 'Boyle point' (also known as $\theta$-point in polymer physics) $\lambda \simeq 1.64$, where $B_{2}=0$ and the system behaves to $\mathrm{O}\left(\phi^{2}\right)$ as an ideal gas. For the smaller particles, the experimental osmotic pressure does not deviate from the van 't Hoff law up to $\phi \simeq 0.15$, which means that the system is very close to the Boyle point, whereas the larger particles yield $-4 \lesssim B_{2} \lesssim-2.4$. Although these values clearly highlight a noticeable attractive dipolar contribution, they quantitatively disagree with what one would predict from the measured values of the coupling constant: for the smaller particles $(\lambda \simeq 0.8$ ) one gets indeed a theoretical value $B_{2}^{\text {teo }} \simeq+3.1$, whereas for the large particles $(\lambda \simeq 1.8)$ $B_{2}^{\text {teo }} \simeq-0.88$. The effective interactions therefore seem to be consistently more attractive than expected. It is also interesting to notice that, for small $\lambda$, the leading term of the effective interaction has the form:

$$
\beta u(r ; \lambda)=u^{\mathrm{hs}}-\frac{\lambda^{2}}{3}\left(\frac{\sigma}{r}\right)^{6},
$$

where $u^{\mathrm{hs}}$ is the HS potential. Note that, in this weak-coupling limit, $u(r ; \lambda)$ decays as $r^{-6}$, like London-van der Waals dispersion forces. Indeed, at variance with the depletion potential we have discussed, $u(r ; \lambda)$ (known in liquid state theory as a 'Sutherland potential') is sufficiently long-ranged to display a stable vapor-liquid coexistence [55], with a critical point at $\lambda_{c} \simeq 2.24, \phi_{c} \simeq 0.2^{12}$. Yet, although this value lies within the virial coefficients experimentally found for the larger magnetite particles, no sign of liquid-liquid separation is observed. Spotting a convincing reason for these discrepancies does not seem to be easy, in particular because more complex effects like the formation of particle chains or columns, which characterize the behavior of dipolar fluid at higher values of the coupling parameter, are not expected to take place.

Dipolar interaction can also be induced by applying for instance an electric field to a dispersion of polarizable particles with no permanent dipole moment. In this case, of course, no orientational average should be performed, for the induced dipoles are all aligned along the external field direction. Preliminary attempts to extract by confocal microscopy the EOS of fluorescent silica particles have been

${ }^{12}$ For $\lambda=\lambda_{c}$, the virial coefficient for the Sutherland potential, which can be analytically evaluated, is $B_{2}=-1.345 B_{2}^{\mathrm{hs}}$, which is in reasonable agreement with the prediction that, for a wide class of attractive potentials, $B_{2}$ $\simeq-1.5 B^{\mathrm{hs}}$ at the critical point [56]. 
made by $\mathrm{Li}$ et al [57] by means of an ingenious setup allowing us to apply electric fields that can be either set along the vertical direction, or made to rotate at $\mathrm{MHz}$ frequency. The field amplitude can moreover be increased up to $150 \mathrm{~V}$ $\mathrm{mm}^{-1}$, which allows us to explore the strong-coupling regime $u(r=\sigma) \gg k_{\mathrm{B}} T$. Unfortunately, although several interesting observations concerning the changes in the EOS and the progressive development of orientational order brought in by the field were made, the investigated system is not sufficiently characterized for any quantitative conclusion to be reached.

As we anticipated, non-spherical particles yield a much richer panorama in terms of phase behavior. A milestone result in condensed matter physics is Onsager's model for the isotropic-to-nematic transition of hard rods that, although rather idealized (it considers only exclude volume effects, and applies only in the limit of very long rods) still yields a fundamental insight into the basically entropic origin of lyotropic liquid crystals. DFT studies of hard rods in the presence of gravity have shown, however, that even for large aspect ratios $r=L / d$, where $L$ and $d$ are the rod length and diameter, deviations from the limiting Onsager's model may strongly influence the equilibrium sedimentation profile [58]. For instance, Onsager's model predicts that the concentration profile in the dense nematic phase is still barometric, but with an exponential decay constant three times larger than $\ell_{\mathrm{g}}$. Conversely, when semi-empirical corrections for higherorder effects are introduced, the nematic packing fraction profile turns out to be approximately linear in $z$ even for $r$ as large as 40. This observation, which highlights once again the exquisite sensitivity of the sedimentation profiles to the EOS of the investigated system, shows that the latter must necessarily be compared with detailed numerical studies of rods with finite aspect ratio. A theoretical and numerical study by Savenko and Dijkstra [59] of the sedimentation behavior of hard spherocylinders with $r=5$ (an aspect ratio already large enough to yield both smectic and nematic mesophases) interestingly shows that the stacking of the different phases along the vertical direction at equilibrium may depend on the height of the sedimentation cell; whereas in a semi-infinite system the most dilute phase at the top of the sediment is always isotropic, this is not the case when $h$ is of the order of a few $\ell_{\mathrm{g}}$, which results in a much more complicated phase diagram.

Experiments had to wait until rod-like particles with controlled aspect ratio and fairly monodisperse size distribution were made available by novel synthesis protocols. By combining confocal microscopy and small-angle x-ray scattering measurements on silica rods, Kuijk et al [60] successfully identified in the equilibrium profile the specific succession of isotropic, nematic and smectic phases predicted for $r$ varying between 3.7 and 8 (see figure 3) ${ }^{13}$. Some discrepancies were nevertheless found with respect to the

13 The experiment actually spanned a moderately large range of Peclét numbers too. Still identifying the quantity ' $a$ ' with the largest microscopic length scale in the problem, for rods in the isotropic dilute phase it is reasonable to put $a \sim L$. With this choice, $P e$ increases from 0.3 for $r=8$ up to 3.4 for $r=3.7$ (the shorter rods are considerably thicker). In the nematic phase, where the rods are strongly interacting, $a$ should rather be taken as the correlation length of the orientational order parameter.

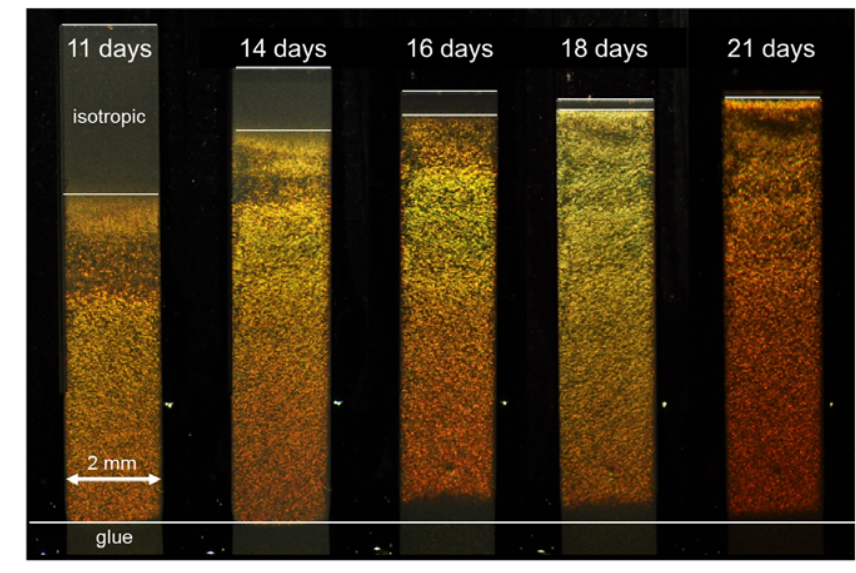

Figure 3. A settling sample of $L=1.9 \mu \mathrm{m}, d=420 \mathrm{~nm}$ rods, illuminated with white light from behind and followed in time. The Bragg-reflecting ordered region that begins to form at the cell bottom grows with time, while the interface between suspension and supernatant descends, until after 20 days the ordered region almost touched the interface. No appreciable changes are subsequently observed. Reprinted with permission from [60]. Copyright 2012, Royal Society of Chemistry.

theoretical phase diagram: in particular, the fully crystalline phase predicted at high volume fraction by simulations [61] is substituted by a smectic-B phase, where long-range orientation order is present in each single smectic layer, but correlations between subsequent layers is lacking. This can either be due to some dispersion in the rod length $(\sim 10 \%)$, or to the presence of residual electrostatic effects (the silica rods are negatively charged). Concerning the latter, a very interesting observation made in [60] is the following. Except at very low values of the ionic strength, the phase diagram for a suspension of charged spheres can generally be mapped onto an HS system, provided that an 'effective', slightly larger particle radius is used. A similar rescaling of the rod diameter (and length) yields a phase diagram that is in reasonable agreement with the simulations for what concerns the phase boundaries, but not for the dependence of the latter on the aspect ratio; hence, using 'effective hard particle' concepts for colloids other than spheres may be misleading. Reference [60], which is still the most informative source about phase equilibria of colloidal rods under gravity, also presents additional valuable information about liquid crystal nucleation and topological defects in the smectic phase. However, a comprehensive experimental analysis of rod concentration profiles, allowing for instance a comparison with detailed theoretical predictions for nematic ordering in the presence of gravity [62], is yet to come.

Together with long rods (fibers), platelets are another kind of particle that, besides finding extensive use in industrial applications, display a complex and only partly understood phase behavior. Wijnhoven et al [63] have studied the competition between sedimentation, gelation, and liquid crystal formation in suspensions of charged colloidal platelets made of aluminum hydroxide (gibbsite) of different sizes, tuning electrostatic forces by the addition of salt. Although the investigation was limited to the identification of different 
phases by polarimetry, a very rich scenario, strongly influenced by the relative strength of gravity, emerged. For instance, the phase behavior of platelets with average diameter $d=210 \mathrm{~nm}$, thickness $L=7 \mathrm{~nm}$, and $\ell_{\mathrm{g}}=1.5 \mathrm{~mm}$ changed drastically by decreasing the solution ionic strength $I$. Whereas for $I=10^{-2} \mathrm{M}$ an isotropic phase lies over a nematic phase, topping in turn an amorphous dense sediment, the phase stacking at $I=10^{-3} \mathrm{M}$ consists of a columnar phase located beneath an isotropic fluid. By further reducing $I$ to $10^{-4} \mathrm{M}$, however, the suspension settles into a single non-birefringent phase, which resembles an amorphous gel. Conversely, much larger platelets with $d=570 \mathrm{~nm}$, thickness $L=47 \mathrm{~nm}$, and gravitational length $\ell_{\mathrm{g}}=29 \mu \mathrm{m}$ always yield an isotropic phase topping an amorphous dense sediment, regardless of the value of $I$. Note that, even for the large platelets, $P e=L / \ell_{\mathrm{g}} \simeq 0.02$; hence, gravity seems to affect delicate phase equilibria even at very low Peclét numbers.

Yet, are the amorphous phases observed for charged platelets at low ionic strength truly akin to the gels generated by spinodal decomposition in depletion systems? Recent surprising observations by Ruzicka et al [64] on suspensions of Laponite, a synthetic clay made of nanometric disks, point to a very different scenario. These suspensions do form quite transparent gels over a typical time of a few thousands hours; however, samples prepared at particle weight concentration $w \lesssim 1 \%$ undergo, after a much longer time (several years) a phase-separation process, witnessed by the appearance of a sharp interface between an upper transparent fluid and a lower turbid gel. Hence, in contrast to gels generated by depletion interactions, where arrest occurs after a phase-separation process has generated strong density fluctuations, here phase demixing takes place in an already formed gel. Conversely,

for $w \gtrsim 1 \%$ the gels remain stable for more than seven years. Very likely, the reason for this puzzling behavior is rooted in the inhomogeneous surface charge distribution on clay particles, which leads to strongly directional interparticle forces. The experimental evidence is indeed strongly reminiscent of what has been theoretically predicted for 'patchy' particles with small valency, namely, capable of forming only a limited number of bonds with other particles [65]. Within this framework, the first dynamic arrest actually corresponds to the formation of a metastable percolating structure. At low $w$, this structure eventually evolves to the equilibrium state by crossing a L-L demixing line, similarly to the case of isotropic shortrange attractions. However, the persistent arrested structures observed for $1 \% \lesssim w \lesssim 2 \%$ should rather be interpreted as an 'empty' liquid, namely, an equilibrium gel structure with

vanishing density, whereas for $w \gtrsim 2 \%$ the suspension rather takes on the structure of a Wigner glass, namely, an arrested structure stabilized by repulsive electrostatic forces. While for a detailed description of the fascinating phase behavior of patchy particles we necessarily have to refer to the seminal theoretical work made by Sciortino and co-workers (see, for instance [65]), in the present context it is however worth stressing that dramatic changes in the sedimentation profile may still take place after astonishingly long (macroscopic) quiescent periods.

\subsection{Gravity effects on soft colloidal solids: gels, foams, and emulsions}

As we have seen, when depletion forces are sufficiently strong to drive a colloidal suspension within the metastable $\mathrm{L}-\mathrm{L}$ coexistence region, a gel-like structure forms. These disordered matrices are soft solids, which in the presence of gravity undergo noticeable restructuring and 'aging' processes that can be accurately followed by sedimentation measurements [66], but which I will unfortunately not be able to cover in this review. Let me just point out that the aging kinetics of colloidal gels can proceed along several routes: for instance, a 'quiescent' stage may or may not precede a collapse of the gel, which in turn can either consist of a slow uniform compression, or of a sudden rupture of the network into clusters that settle fast and eventually compact into an arrested denser structure. Interestingly, if collapse proceeds along this latter route, the gel eventually reaches a steadystate profile whose shape neither depends on the strength of the attractive forces, nor on the initial particle volume fraction [67]. In this case, equation (7) rather yields the dependence on $\phi$ of the gel elastic modulus $\sigma$, which is found to obey, at least up to $\phi \simeq 0.5$, a power law $\sigma=A \phi^{\alpha}$ with $\alpha \simeq$ 4 , in reasonable agreement with recent simulations of a 'multi-mode' plastic consolidation of the gel [68].

Depletion gels, however, are just a kind of disordered solid structures that colloidal suspensions and soft materials can form; other examples of noticeable practical importance are foams and concentrated emulsions, namely, structures made by tightly packed drops or bubbles surrounded by an immiscible fluid. Suppose that, by means of an osmotic membrane, permeable only by the surrounding continuous phase, we compress a dispersion of drops or bubbles until they reach close packing, which corresponds to a volume fraction $\phi_{c}=\phi_{\text {ocp }}$ if the bubbles or the drops are monodisperse in size and arranged in a crystalline structure and, when the packing is fully disordered, to a value close to (but arguably lower, if

dynamic arrest into a glass phase occurs) $\phi_{c} \lesssim \phi_{\mathrm{rcp}} \simeq 0.64$ (random close packing). Compressing the system beyond this limit requires us to apply a force per unit area to the membrane equal to the stress associated with the contact network throughout the dispersion; this elastic stress $\Pi$ plays

a role that is fully analogous to the osmotic pressure for a dispersion of free Brownian particles, although it is usually orders of magnitudes larger than the latter. More precisely, we can write $\Pi=\gamma \mathrm{d} A / \mathrm{d} V$, where $\gamma$ is the interfacial tension

between the two immiscible fluid phases, and $\gamma \mathrm{d} A$ is the work required to increase the interfacial area of the dispersion by $\mathrm{d} A$, by extracting a volume $\mathrm{d} V$ of the continuous phase. If $\gamma$ is moderately low and the emulsion/foam is made to settle in a vessel sufficiently tall to yield a substantial compression, the integration of the concentration profile $\phi(z)$ induced by gravity (again acting as a 'generalized membrane') allows us to reconstruct the elastic modulus $\Pi(\phi)$ of the system (note that this experimental opportunity was pointed out by Princen

[69] even before the same idea was used for colloidal

suspensions). In fact, the concentration profile is again a function of the scaled variable $z / \ell_{\mathrm{g}}$, where the relevant 'gravitational length' $\ell_{\mathrm{g}}$ is here given by the ratio $l_{\mathrm{c}}^{2} / a$ of the square of the capillary 


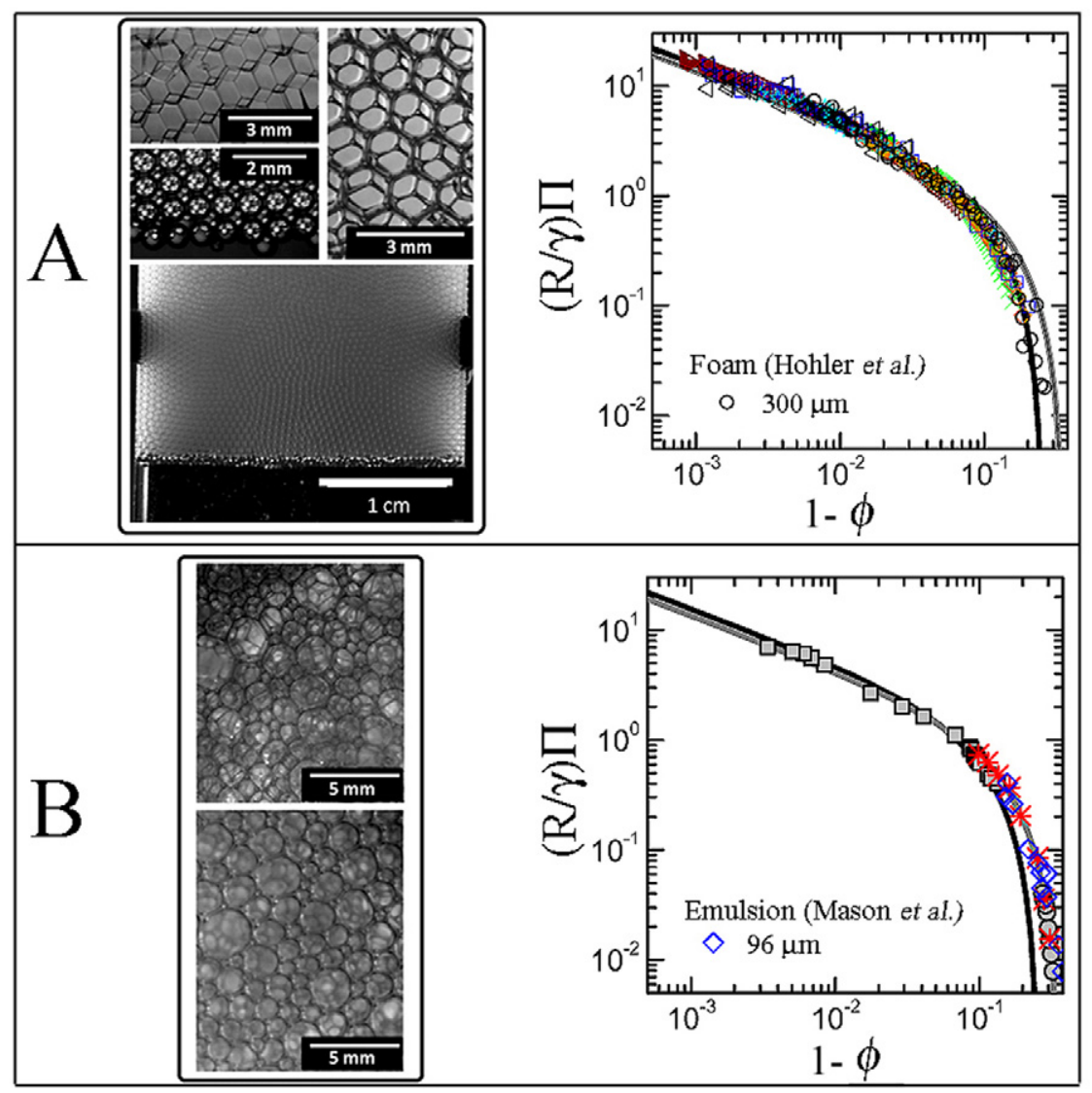

Figure 4. Foam structures and osmotic pressure at settling equilibrium for monodisperse, ordered samples (panel A) and polydisperse, disordered samples (panel B), also including results obtained by rheological methods [70] or by membrane compression of foams produced by microfluidic flow-focusing [71]. The solid lines correspond to equation (12), with parameters $k$ and $\phi_{c}$ for monodisperse and polydisperse samples discussed in the text. Images and data plots reprinted with permission from [72]. Copyright 2013, Royal Society of Chemistry.

length $l_{\mathrm{c}}=(\gamma / \Delta \rho g)^{1 / 2}$ to the droplet size $a^{14}$. Although it is possible to show rigorously that, in the asymptotic limit $\phi \rightarrow$ $1, \Pi \sim(\gamma / a)(1-\phi)^{-1 / 2}$, no theoretical prediction exists for the behavior of $\Pi(\phi)$ over the whole $\phi$-range. Nevertheless, the experimental evidence for ordered monodisperse foams suggests that, at least for $\phi$ sufficiently larger than $\phi_{c}, \Pi(\phi)$ is very well approximated by

$$
\Pi(\phi)=k \frac{\gamma}{a} \frac{\left(\phi-\phi_{c}\right)^{2}}{\sqrt{1-\phi}},
$$

where $k$ is a dimensionless constant. The accuracy of this empirical expression has recently been confirmed by extensive equilibrium sedimentation measurements [72]. In fact, equation (12) describes a wide class of aqueous systems (see figure 4), including monodisperse and polydisperse foams and emulsions of diverse droplet sizes, with a parameter $k$ which seems to depend only on whether the packing is ordered $(k \simeq 7.3)$ or random $(k \simeq 3.2)$.

The concentration profiles were obtained by measuring the electric conductivity of the system as a function of the vertical position $z$. This may seem a rather dubious method, for one may expect the current to spread through a large

${ }^{14}$ A simple algebraic manipulation shows that, in the expression for $\ell_{\mathrm{g}}$, the role of $k_{\mathrm{B}} T$ is then taken by $E / 3$, where $E$ is the total interfacial energy of a spherical droplet of radius $a$. region of the sample, but it is actually a clever choice, because the largest contribution to the total resistance is given by the sample region very close to the electrodes; in fact, the vertical resolution is basically limited by the electrode size [72]. In their very interesting paper, Maestro et al [72] also manage to quantitatively account for the empirical values found for $k$ in the two different cases, and also propose a modified version of equation (12) that, besides capturing the behavior for $\phi \rightarrow \phi_{c}$ too, agrees with most existing experimental data for ordered foams and emulsions. This simple general behavior shared by disordered solids stabilized by surfactants does not seem to be sufficient, however, to describe the osmotic pressure of concentrated particle-stabilized emulsions. Indeed, recent centrifuge sedimentation measurements [73] suggest that $\Pi(\phi)$ is strongly influenced by the effective repulsion between adsorbed particles stemming from the deformation of the liquid-liquid interface between particles on neighboring droplets; squeezing the interdroplet layer indeed entails a substantial energy cost. Due to the important technological role of Pickering emulsions, further studies in this direction would be extremely interesting.

\subsection{Living with weight: sedimentation of active particles}

At variance with what the Greek name suggests, plankton includes a large number of microscopic species that are not just 
idle 'wanderers' carried around by marine currents, but rather motile organisms, which can perform extensive diurnal vertical migration. For this and several other reasons, scrutinizing the behavior of micro-swimmers in the presence of gravity is particularly relevant to the field of marine ecology. Living organisms are however in a perennial non-equilibrium state (or, at least, insofar as they live), hence the simplest experimental condition we can think of is a steady state. It is then wise to approach the subject cautiously, by starting with artificial systems that at least mimic some aspects of the behavior of living microorganisms: an interesting example are the 'active' Brownian particles that self-propel by generating a chemical potential gradient in the solvent, currently among the most investigated system in soft matter. This can be accomplished by using 'Janus' particles, displaying in their structure two distinct regions with different surface properties, obtained for instance by coating a fraction of the surface of a standard colloidal polymer sphere with a thin platinum layer; when the particles are immersed in a mixture of water and hydrogen peroxide, the platinum layer catalyzes the decomposition of $2 \mathrm{H}_{2} \mathrm{O}_{2}$, which takes place only on one side of the particle, driving it toward the region where oxygen is produced.

By this locomotive power, the particles move with a steady-state velocity of constant amplitude $v_{a}$ (which grows with the concentration of the $\mathrm{H}_{2} \mathrm{O}_{2}$ 'fuel'), but whose direction is progressively randomized by the thermal reorientation of the particle axis on a time scale set by the Brownian rotational diffusion time $\tau_{\mathrm{r}}=8 \pi \eta a^{3} / k_{\mathrm{B}} T$. For $t \gg \tau_{\mathrm{r}}$ the particle motion becomes then diffusive, but with an effective diffusion coefficient $D^{*}=D_{0}+\tau_{\mathrm{r}} v_{a}^{2} / 6$ which is much larger than the bare diffusion coefficient $D_{0}{ }^{15}$. On the basis of our former considerations, a dilute suspension of these active particles may then be supposed to generate in the presence of gravity a barometric sedimentation profile, but with a much larger effective gravitational length $\ell_{\mathrm{g}}^{*}=\ell_{\mathrm{g}} D^{*} / D_{0}$, which can be tuned by varying the fuel concentration. This has been confirmed by Palacci et al [74] using an ingenious microfluidic device, where permeable gel microstructures provide steady $\mathrm{H}_{2} \mathrm{O}_{2}$ supply and $\mathrm{O}_{2}$ waste removal. By changing the $\mathrm{H}_{2} \mathrm{O}_{2}$ concentration, $\ell_{\mathrm{g}}^{*}$ was varied between 6 and $21 \mu \mathrm{m}$. It is convenient to define an 'effective' Péclet number

$$
P e^{*}=\frac{a}{\ell_{\mathrm{g}}^{*}}=\frac{P e}{1+(2 / 9) P e_{a}^{2}}
$$

which combines the sedimentation Péclet number with the Péclet number $P e_{a}=a v_{a} / D_{0}$ associated to the active motion. For the particles with $a=1 \mu \mathrm{m}$ used in the experiment, $0.05 \lesssim P e^{*} \lesssim 0.17$, although in the absence of active motion $P e \simeq 5.5$, the particles may therefore be regarded de facto as Brownian over the whole investigated propulsion range $0.3 \mu \mathrm{m} \mathrm{s}^{-1} \leqslant v_{a} \leqslant 3 \mu \mathrm{m} \mathrm{s}^{-1}$. It is also useful to point out that gravity should in principle lead to a partial alignment of the suspension, with the mean swimming direction oriented against the gravitational field [75]. This peculiar effect, generated by the interplay between self-propulsion and gravity

${ }^{15}$ Although it is tempting to define an 'effective temperature' $T^{*}=k_{\mathrm{B}}^{-1} f D^{*}$ we must recall once again that the active system is not at therma equilibrium;

for instance, the particle velocity distribution is far from being Maxwellian! (which however does not affect the swimming direction directly) should also induce a larger accumulation of the particles at the cell bottom than expected from the barometric law, as indeed observed in [74].

What about true living organisms, such as bacteria?We should first point out that bacterial swimming is rather different from the ballistic motion, progressively randomized by Brownian rotation, we have identified for self-propelling particles. In many cases, it can rather be regarded as consisting of sudden changes of the direction of the velocity $v_{a}$, taking place after an average time lapse $\tau$ : this idealized mechanism yields the so-called the 'run-and-tumble' (R\&T) mode ${ }^{16}$. Nevertheless, it can be shown that, in the absence of 'environmental' factors that cause the propulsion velocity or the tumbling time to vary in space (such as an inhomogeneous distribution of nutrients in the solvent, or collective effects depending of the local bacterial concentration), a R\&T motion can always be mapped onto a (suitably chosen) random walk [76]. This however does not apply even when the motility is not isotropic, which is for instance the case of sedimentation, where upward and downward swimmers have different speeds $v_{a} \pm v_{s}\left(v_{s}\right.$ being as always the Stokes velocity). For a dilute suspension of noninteracting bacteria, a one-dimensional model of this R\&T biased diffusion process still leads to a barometric profile, but with a gravitational length given by

$$
\ell_{\mathrm{g}}^{*} \frac{v_{a}^{2}-v_{s}^{2}}{v_{s}} \tau \text {. }
$$

Note that, whereas for standard Brownian particles the gravitational length vanishes only for $v_{s} \rightarrow \infty$ (for instance in an ultracentrifuge at very large speed), here $\ell_{\mathrm{g}}^{*}=0$ for a finite value of $v_{a}=v_{s}{ }^{17}$. The physical interpretation of this rather peculiar result is simple: if the bacteria cannot swim upstream faster than $v_{s}$, they will never be able to generate a diffuse barometric region, and the steady-state profile is flat. This is usually not the case for natural sedimentation, since in general $v_{s} \ll v_{a}$, but can be easily be obtained by forced sedimentation in a centrifuge.

Several other surprises may be expected by considering the settling behavior of concentrated bacteria suspension, and even more if we take into account an important feature of bacteria which is not shared by artificial swimmers: they breed. For instance, numerical simulations suggest that, by increasing the bacterial growth rate, the system could make an abrupt transition from a sedimentation regime in which the density profile is barometric to a uniform growth-dominated regime where the density is spatially quasi-uniform [78]. Unfortunately, from the experimental side this intriguing world of bacterial sedimentation is so far mostly unexplored. Of course, settling experiments on bacteria are hard to perform, in

${ }^{16}$ As a matter of fact, the tumbling time $\tau$ of bacteria is often of the same order of magnitude as their rotational diffusion time $\tau_{\mathrm{r}}$. A simple reason for this evolutionary adaptation is nicely discussed in [76].

17 The functional form of $\ell^{*}$ in 3D is more complicated, but one still finds $\ell^{*}$ $=0$ for $v_{a}=v_{s}$. This result is slightly modified if hydrodynamic interactions (HI) between bacteria are taken into account [77]. However, this is a weak effect that just slightly 'softens' the profile for $v \simeq v_{s}$, because these fluctuations are much shorter-ranged than those generated by the settling process we shall later discuss [76] 
particular when they require long time, and require stringent controls to make sure that other driving forces, for instance of chemotactic nature, are not at work: but, if carefully performed, they might be extremely rewarding.

\subsection{A largely uncharted world}

It is rather baffling that equilibrium sedimentation studies of macromolecules and biological fluids still fill a small niche in the vast field of analytical centrifugation, given its extensive use in chemistry, biology, and medicine. Most studies have indeed aimed to investigate, from the dilute barometric profile, protein solvation or association effects, the latter being particularly relevant for studying complexes of membrane proteins with the surfactants used to solubilize them [5]. Although a limited number of investigators have made substantial and creditable efforts to investigate interparticle interaction effects by extracting virial coefficients [79], or by using the so-called 'tracer equilibrium' method ([80], see also section 4), the opportunity of reconstructing the full EOS and phase behavior from sedimentation measurements has not been picked up by the community dealing with ultracentrifugation, at least to my knowledge.

Admittedly, basic concepts in colloid science like the potential of the mean force become a bit blurred in sedimentation studies of biological fluids, for these suspensions often contains small molecular components whose sedimentation rate is not negligible: hence, settling makes the solvent inhomogeneous, and 'volume' terms in the effective interactions surely become relevant. But they are surely worth the effort, because equilibrium sedimentation measurements with centrifuges are not only feasible, but surely less timeconsuming than in natural gravity. Too see this, we should first notice that what really fixes the experimental scene in sedimentation studies is the value of $\ell_{\mathrm{g}}$, which should be large enough to allow for a good measurement of the barometric profile (checking the theoretical value for $\ell_{\mathrm{g}}$ is always a good habit), but not so large that the volume fraction at the cell bottom is still small. For instance, in the inset of figure 1 we see that the fluid region in the HS profile spans about $20 \ell_{\mathrm{g}}$ : hence to get a fully developed profile the minimal cell height $h_{\text {min }}$ must be at least, say, $\sim 30 \ell_{\mathrm{g}}$. Fixing an accessible range for $\ell_{\mathrm{g}}$ has two consequences for forced sedimentation studies.

- Speed: The absorbance or interferometric setup of a highperformance analytical ultracentrifuge typically allows us to detect simultaneously 1000-2000 points of the profile along the radial direction, with a resolution that ranges between $10--100 \mu \mathrm{m}$; hence, a value of $\ell_{\mathrm{g}}$ between, say, 0.1 and $1 \mathrm{~mm}$ would surely allow us to cover the whole sedimentation profile still retaining a good resolution in the barometric region. Consider then a small protein like lysozyme, with a buoyant mass $m \simeq 6.8 \times 10^{-21} \mathrm{~g}$ : if we wish $\ell_{\mathrm{g}}=1 \mathrm{~mm}$, we must provide an (average) acceleration of the order of $6 \times 10^{4} \mathrm{~g}$, which is far below the maximum value for an ultracentrifuge, making equilibrium sedimentation measurements fully feasible. However, ultracentrifuges are not usually meant to work with acceleration lower than about 700-1000 g, and this sets an upper limit for the particle size that can be measured.

- Duration: Fixing $\ell_{\mathrm{g}}$ also sets a lower limit for experimental time scale $\Delta_{t}>h_{\min } / v_{s}=30 t_{\mathrm{g}}$, where $t_{\mathrm{g}}=$ $\ell_{\mathrm{g}} / v_{s}=t_{s} / \mathrm{Pe}$ is the time it takes for a dilute solution to settle by $\ell_{\mathrm{g}}$; in practice, as we shall shortly see, $\Delta_{t}$ should be at least 5-10 times larger. However, we simply have

$$
t_{\mathrm{g}}=\frac{\ell_{\mathrm{g}}^{2}}{D_{0}}=f \frac{k_{\mathrm{B}} T}{\ell_{\mathrm{g}}}=C \frac{k_{\mathrm{B}} T}{\ell_{\mathrm{g}}} \eta a,
$$

where $C$ ( $=6 \pi$ for spheres $)$ is a just a shape factor. Hence, at fixed $\ell_{\mathrm{g}}, \Delta_{t}$ is linearly proportional to the particle size (but does not depend on the density mismatch with the solvent). This means for instance that the experiment described in figure 1, if performed with HS the size of lysozyme, would take days instead of months (which of course may still be a long booking period for an ultracentrifuge).

Equilibrium sedimentation measurements in ultracentrifuge may then allow a largely uncharted world to be entered and to address several questions concerning the nature of the interaction potential between biological macromolecules. The latter are known for instance to be extremely salt-specific, which is one of the most striking manifestations of the still partly mysterious 'Hofmeister effect' [81]. Even more stimulating is the sedimentation of structures originating from self-aggregation, the paradigmatic case being that of surfactant micelles. Back in 1993, Duyndam and Odijk pointed out that, due to the interplay between sedimentation and aggregation, the equilibrium concentration profile of dilute solutions of surfactants that aggregates in the form of rod-like micelles should not be barometric (either in the exponential form proper of gravity settling, or with the slightly more complex shape obtained in a centrifuge), but rather of the form $\phi(z)=a\left(1+b z / \ell_{\mathrm{g}}\right)^{-2}$, where $a$ and $b$ do not depend on $z$ [82]. More generally, little has been theoretically assessed concerning collective effects on the shape of micellar aggregates; hence, general investigations of the osmotic EOS of surfactant solutions, so far totally lacking, would be extremely useful.

Several proteins of great biological relevance are also known to spontaneously aggregate into large structures, the most important of them probably being tubulin, a small globular protein dimer that polymerize into microtubules, those long hollow filaments with an external diameter of just $25 \mathrm{~nm}$ and up to tens of micrometers long, that constitute, together with actin filaments, the basic structure of the cytoskeleton of eukaryotic cells. Being so long, microtubules organize into a nematic phase at very low concentration: yet, even natural gravity seems to play a role in their phase behavior, for in microgravity conditions no sign of the nematic order displayed in ground experiments is apparently observed [83]. Prompted by this puzzling result, Baulin has tried to develop a general analysis of self-assembled aggregates in the gravitational field [84], which moves along a line similar to the approach in [82], but also takes into account the finite flexibility of the aggregates, which are then described similarly to those reversible worm-like surfactant aggregates known as 'living 
polymers' [85]. The analysis generates several surprises. Writing $\epsilon_{N}=\epsilon_{\infty}+\delta / N$ for the energy (in $k_{\mathrm{B}} T$ units) of a tubulin dimer in an aggregate of $N$ dimers [84] (where the excess contribution $\delta>0$ promotes the formation of aggregates), and calling $\phi_{0}$ the total volume fraction at the cell bottom, the sedimentation profile for non-interacting ideal aggregates is found to be

$$
\phi(z)=\frac{\phi_{0}}{\left[1+\sqrt{\phi_{0} \mathrm{e}^{\delta}} z / \ell_{\mathrm{g}}\right]^{2}},
$$

which strictly parallels the result in [82]. However, for finite concentration (but still in the isotropic phase), if one takes into account excluded volume interactions at the level of the second virial coefficient in Onsager's limiting theory, the profile shows a linear dependence on $z, \phi(z)=\phi_{0}-(2 / \pi) z / \ell_{\mathrm{g}}$. The transi $\mathrm{n}$ between the two regimes takes place at a position tio

$z_{c} \sim \sqrt{ } \phi h \ell_{\mathrm{g}}$, where $h$ is the initial height, which is generally very close to the top of the profile. The role of gravity is better highlighted by considering the distribution of the aggregates over the whole cell. Whereas low gravitational fields just induce a redistribution of aggregates with height and do not influence the micellar equilibrium properties, sufficiently strong coupling consistently increases the average micellar aggregation number. This means that integrating the concentration profile we would not get the equilibrium EOS. Baulin points out that this might often be the case for microtubules even in natural gravity where, even with a container height of the order of $1 \mathrm{~cm}, h / \ell_{\mathrm{g}}$ can be ten times larger than the value for strong coupling to set in. Again, this rich and unusual scenario is still experimentally unexplored.

\section{Settling dynamics}

The brilliant strategy developed by McMillan and Mayer [12], allowing us to trace out the degrees of freedom of the molecular components, works only at equilibrium. In colloid dynamics, unfortunately, the neglected solvent peremptorily peeps out. In its Brownian wandering, a particle stirs the surrounding fluid, generating a velocity field that affects the motion of the other particles, which of course return the favor. Whereas they do not affect any structural properties at equilibrium, ${ }^{18}$ these HI play a crucial and, as we shall shortly see, rather wicked role in determining transport properties [86]. In particular, the kinetics of sedimentation is probably one of the subjects where statistical mechanics, fluid dynamics, and liquid state physics are more intimately connected, generating surprising and challenging effects that are still partly understood. Because of this, we shall linger only briefly on this track, just to get a taste of the perilous world of settling dynamics (for a detailed introduction, see [87]).

\subsection{Hindered settling and the Smoluchowski paradox}

As we already mentioned, the settling speed of an isolated particle (the 'Stokes velocity') can be easily written in terms

${ }^{18}$ It is useful to stress, however, that HI do matter for active particles even at steady state, as clearly discussed in [76] of low Reynolds number ${ }^{19}$ expression for the hydrodynamic friction coefficient as $v_{s}=m^{*} g / f$. Since, for a particle of characteristic size $a, m^{*} \propto \Delta \rho a^{3}$ and, for Re $\ll 1, f$ scales as $\eta a, v_{s} \propto(\Delta \rho g / \eta) a^{2}$ (for a sphere $\left.v_{s}=2 \Delta \rho g a^{2} / 9 \eta\right)^{20}$. For a particle suspension, however, the problem is much harder, even at very small volume fraction, because of HI. Without entering into the detail of this really complex subject (for a very good review, see [86]), I shall give a simple account of those physical aspects of this issue that will be useful in what follows, forgetting for the moment about Brownian motion. At low $\mathrm{Re}$, and in stationary conditions, the velocity field $\boldsymbol{u}$ of an incompressible fluid $(\boldsymbol{\nabla} \cdot \boldsymbol{u}=0)$ is governed by the Stokes creeping-flow equation: $\eta \nabla^{2} \boldsymbol{u}-\nabla p+\boldsymbol{f}=0$, where $\boldsymbol{f}$ is the external force per unit volume. If we subtract out from $p$ the direct effect of gravity on the fluid, $-\rho_{0} \boldsymbol{g} \cdot \boldsymbol{r}$ (hence regarding $p$ as a 'dynamic pressure'), the external forces are only due to the stresses exerted by the settling particles of the fluid. For a single point-like particle in $\boldsymbol{r}_{i}$ we can write $\boldsymbol{f}=\boldsymbol{F} \delta\left(\boldsymbol{r}-\boldsymbol{r}_{0}\right)$, and the solution for $v(r)$ is just the Green function of the Stokes equation: $\boldsymbol{u}(\boldsymbol{r})=\mathbb{T}\left(\boldsymbol{r}-\boldsymbol{r}_{0}\right) \cdot \boldsymbol{F}$, where

$$
\mathbb{T}(\boldsymbol{r})=\frac{1}{8 \pi \eta r}\left(1+\frac{\boldsymbol{r} \boldsymbol{r}}{r^{2}}\right)
$$

is called the Oseen tensor, and we have also assumed $\boldsymbol{u}(\infty)$ $=0$. Due to the linearity of the Stokes equation, the velocity field generated by an arbitrary distribution of point-forces can be obtained by superimposing these basic solutions, usually called Stokelets. In particular, the 'disturbance' flow generated by a particle with finite size and arbitrary shape can be obtained by integrating over the particle surface $S$ the Stokelets generated by the elementary forces $\boldsymbol{f}\left(\boldsymbol{r}_{i}\right) \mathrm{d} S=$ $-\boldsymbol{\sigma}\left(\boldsymbol{r}_{i}\right) \cdot \boldsymbol{n}\left(\boldsymbol{r}_{i}\right) \mathrm{d} S$, where $\boldsymbol{\sigma}\left(\boldsymbol{r}_{i}\right)$ is the local stress ${ }^{21}$. The explicit calculation is prohibitive, but a multipole expansion shows that, provided that an external force acts on the particle, ${ }^{22} \boldsymbol{u}(\boldsymbol{r})$ still vanishes as $r^{-1}$ far from the particle.

The 'disturbance' generated by the particle is then very long ranged, and this has important consequences. Consider just two isolated spheres: still neglecting Brownian motion, one finds that the settling velocity of each sphere increases with respect to $v_{s}$, and also depends on the orientation of the vector $r$ connecting the particle centers with respect to $\boldsymbol{g}$, being maximal when $\boldsymbol{r} \| \boldsymbol{g}$, minimal when $\boldsymbol{r} \perp \boldsymbol{g}$, and in general oblique with respect to the vertical. But the velocity

is the same for both spheres, so the particles neither come closer or farther, nor change their orientation: they behave as a

${ }^{19}$ Even when the Peclét number is large, colloid sedimentation usually takes place at low Reynolds number. Indeed $R e=\left(D_{0} / v\right) P e$ and, even for very small particles, the fluid kinematic viscosity $v \gg D_{0}$.

${ }^{20}$ In general, the friction factor on an object of maximal linear dimension $L$ is not very different from that of a sphere of diameter $L$. Of course, for anisotropic particles, $f$ depends on the direction of motion with respect to the particle orientation. For long rods, for instance, the ratio $f_{11} / f_{\perp}$ between the friction coefficients for motion along and perpendicularly to the symmetry axis is about $1 / 2$. This value for $v_{s}$ applies to settling in an infinite container; close to a side wall, $v_{s}$ can be very different.

21 This also allows us to evaluate the drag force and torque via general results known as Faxén laws [86].

${ }^{22}$ In the case of active particles, which move using only internal forces with null resultant, the velocity field decays at least as a dipole, and does not yield the subtle convergence problems we shall discuss. 
'rigid dumbbell' connected by a 'phantom hydrodynamic rod'. Conversely, if we consider three spheres, the configuration does change. For instance, if the particles are initially aligned along the horizontal, the central one falls faster: two-body forces are then inadequate to describe this behavior. Hence HI: (i) are intrinsically multi-body; (ii) perturb the equilibrium structure of a suspension, at least when $P e \gg 1$. But the real bad news comes when considering an infinite number of particles, for the resulting disturbance generated by monopole $1 / r$ fields diverges: as a matter of fact, the average settling velocity $\langle\boldsymbol{v}\rangle$ of a particle subjected to this total disturbance diverges too, an apparently paradoxical result first pointed out by Smoluchowski [88].

However, real sedimentation experiments are not performed on an infinite fluid at rest, but in a vessel that is bounded by a bottom wall ${ }^{23}$. Let us then forget for the moment about $\mathrm{HI}$, and recall that $v_{s}$ is the single-particle settling speed with respect to the fluid, and not to the vessel. In the presence of a wall at the container bottom, the average friction force $\langle\boldsymbol{F}\rangle$ exerted by the particles on the solvent generates a pressure gradient ${ }^{24} \nabla p=n\langle\boldsymbol{F}\rangle$ driving a backflow with average speed $u(\phi)$ of the fluid against $g$ that, for an incompressible fluid, can be found by volume conservation: $\phi v(\phi)+(1-\phi) u(\phi)=0$. Neglecting HI amounts to state that each particle settles with $v_{s}=v(\phi)-u$ respect to the fluid, which, using volume conservation, yields $v(\phi)=(1-\phi) v_{s}$. Hence, the settling speed decreases with $\phi$ : this effect, due to solvent backflow, is known as hindered settling. Taking into account that $\mathrm{HI}$ is far from being trivial; in fact, it is only through a mathematical tour de force that Batchelor managed to get rid of spurious divergences and to prove that, for a dilute HS system, the solvent backflow 'renormalizes' the average settling velocity $v(\phi)=\langle v\rangle$ to a value given by [89]

$$
v(\phi)=v_{s}\left[1-6.55 \phi+\mathrm{O}\left(\phi^{2}\right)\right]
$$

which corresponds to a much stronger hindered settling effect $^{25}$. It is important to point out that Bachelor's result stems from a purely deterministic hydrodynamic calculation, which neglects thermal fluctuations: however, it can be shown that Brownian agitation does not influence $v(\phi)$, which is fully determined by long-range hydrodynamic effects [90]. Actually, Batchelor result does not necessarily work for strongly non-Brownian particles, because, as we anticipated, the suspension structure factor at high $P e$ may differ considerably from the equilibrium one, even at low $\phi$. As a matter of fact, a thorough theoretical analysis

${ }^{23}$ Smoluchowski was fully aware that this paradox applies only to unbound settling, and discusses in this anticipating paper, which can be freely downloaded from www.mathunion.org/ICM/ICM1912.2, several issues concerning backflow through disordered and ordered particle arrangements.

${ }^{24}$ Rigorously justifying this pressure gradient is not easy, and requires the use of an 'effective' Stokes equation, obtained by ensemble-averaging the contributions of each single particle [86]

25 This result is obtained by approximating the HS radial distribution function to a Heaviside step function, $g(r)=\theta(r-2 a)$. In fact, a large par of the fluid in the region $a<r<2 a$, inaccessible to the other particles, is dragged down by each sphere because of stick boundary conditions. This is accompanied by an equal upward flux in the fluid volume that is accessible to a test sphere, which yields an additional solvent backflow equal to $-4.5 v_{s}$ $\phi$. HI just provide the remaining $-1.55 \phi$ contribution. of dilute, fully non-Brownian suspensions by Cichocki and Sadlej [91], supported by numerical simulations [92], yields $v(\phi)=v_{s}(1-3.87 \phi)$. This strong reduction of hindered settling effects is mostly due to the zero-wavevector behavior of the structure factor $S(0)=1-1.64 \phi$, witnessing a strong increase of the probability for two particles to be in contact with respect to the equilibrium structure (where $S(0)=1-$ $8 \phi)$. Simulation and theoretical results for $g(r)$ and $S(q)$ at low volume fraction are contrasted to their equilibrium values in figure 5.

In a following paper [93], Batchelor managed to extend his results to the case of spheres that, on top of excluded volume forces, interact with forces having a range much shorter than the HS diameter $\sigma$, obtaining, in terms of the second virial

coefficient $B_{2}^{*}=B_{2} / B_{2}^{\text {hs }}$ normalized to the HS value:

$$
\frac{v(\phi)}{v_{s}}=1-\left[6.55-3.52\left(1-B_{2}^{*}\right)\right] \phi+\mathrm{O}\left(\phi^{2}\right),
$$

which for sticky HS becomes (see footnote on p 6) $v(\phi) \simeq v_{s}$ $\left[1-\left(6.55-0.89 \tau^{-1}\right) \phi\right]$. Note that, for $B^{*}<-2.86$,

which is still quite a bit larger than the critical point value $B_{2}^{*} \simeq-1.5$, the settling velocity actually grows with $\phi$. In the opposite case of very long-ranged repulsive potentials, such as weakly screened electrostatic interactions, the behavior can however be very different. A strong electrostatic repulsion is evidenced by a strong peak in the structure factor, marking the existence of a well-defined value $R$ for the interparticle separation. As clearly pointed out by Batchelor [89], in the presence of this additional length scale (besides the particle size $a$ ), the reduction with respect to the Stokes speed must scale as $v(\phi)-v_{s} \propto-v_{s} a / R$ : since $a / R \propto \phi^{1 / 3}$, this suggest a non-analytical behavior for the settling velocity $v(\phi)=v_{s}(1$ $\left.-p \phi^{-1 / 3}\right)$, where $p$ is a positive constant. Such a trend for $v(\phi)$, which can be rigorously derived for an ordered array of settling spheres, seems to be approximately obeyed by strongly de-ionized silica spheres in ethanol, still too dilute to crystallize [94]: this shows once again that, for unscreened electrostatic interactions, the concept of 'dilute suspension' makes little sense ${ }^{26}$.

Extending the former results much beyond first or second order in $\phi$ is very hard: Figure 6 shows that, for HS, even very sophisticated analytical approaches still fail to properly fit the experimental data either at low [95] or high [96] $\phi$, which are conversely quite well described by numerical simulations [97]. Rather curiously, the same data are reasonably well approximated by the simple empirical expression [87] $v(\phi)=$ $v_{s}(1-\phi)^{\alpha}$, with a best fit exponent $\alpha$ that is just $10 \%$ smaller than the value $\alpha=6.55$ required for consistency with the Batchelor low- $\phi$ result (which may be due to residual dispersion forces $)^{27}$. Writing $H(\phi)=v(\phi) / v_{s}$,

\footnotetext{
${ }^{26}$ Electrokinetic effects on the settling velocity of a single charged particle due to double-layer relaxation (related to the so-called sedimentation potential), are generally weak unless the screening length is comparable to the particle size. Besides, telling them apart for the latter is far from being easy.

${ }^{27}$ Note that the different expression $v(\phi)=v_{s}\left(1-\phi / \phi_{0}\right)^{6.55 \phi_{0}}$, where $\phi_{0} \simeq$ 0.64 is the random close packing (RCP) for HS, which is sometimes alternatively used, gives a much poorer fit to the data. Actually, this expression is conceptually incorrect, for it implies that no backflow takes place through spheres at close packing. In this limit, the model describes in fact the equivalent situation of a fluid permeating a disordered solid.
} 

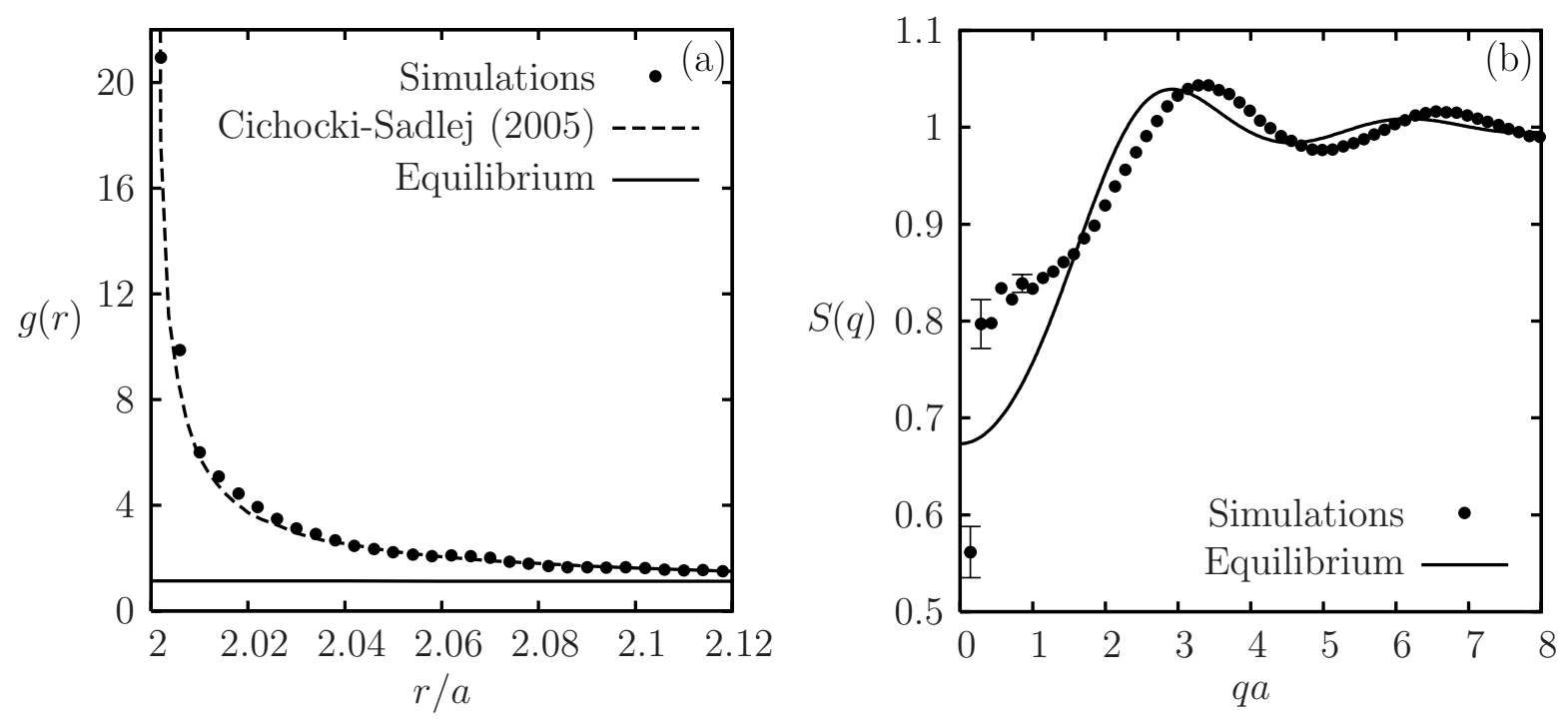

Figure 5. Numerical results for the pair correlation function $g(r)$ (left) and for the structure factor $S(q)$ (right) for fully non-Brownian particles $(P e=\infty)$ at steady sedimentation state, compared with their equilibrium counterparts and to the theoretical results in [91], for $\phi=0.05$. Reprinted with permission from [92].

we see that for HS the factor $H(\phi)$, accounting for HI effects on the friction coefficient, decreases with $\phi$ monotonically. This is not necessarily true in the presence of additional contributions to the interaction potential: for instance, numerical simulations [98] indicate that, for sufficiently strong short-ranged attractions $\left(B^{*}<-0.86\right), H(\phi)$ first increases

as predicted by equation (17), but then reaches a maximum for $\phi \simeq 5 \%$ and finally decreases at larger $\phi$. The physics of this non-monotonic behavior can be simply grasped as follows: the tendency to particle clustering brought in by the attractive interactions, which increases $v$, is eventually overcome at large $\phi$ by the overwhelming hindering caused by hydrodynamic interactions ${ }^{28}$. Notably, this non-monotonic behavior disappears, at least for stable suspensions $\left(B_{2} \gtrsim\right.$ $\left.-1.5 B_{2}^{\text {hs }}\right)$, when $P e \gtrsim 8$, confirming the strong effect of the Peclét number on $S(q)$ [100].

Before describing the time-evolution of the settling concentration profile, it is also useful to recall that $\mathrm{HI}$ modifies the diffusion coefficient too. The latter, however, is also influenced by direct interparticle forces: in fact, the hydrodynamic limit of the collective diffusion coefficient is given by the generalized Stokes-Einstein relation:

$$
D(\phi)=D_{0} \frac{H(\phi)}{S(0)}=V_{\mathrm{p}} \frac{\partial \Pi}{\partial \phi} \frac{H(\phi)}{f}=\frac{1}{g \Delta \rho} \frac{\partial \Pi}{\partial \phi} v(\phi),
$$

where, again, $V_{\mathrm{p}}$ is the particle volume, $f$ the single-particle friction coefficient, and $S(0)$ the structure factor at $q=0$ (which of course depends on $\phi$ ). Since for a dilute suspension of (Brownian) HS $S(0)=1-8 \phi+\mathrm{O}(\phi)^{2}$, the concentration dependence of $D$ is given at order $\phi$ by $D(\phi)=D_{0}(1+$ $1.45 \phi)$. Note that, at variance with $v(\phi), D(\phi)$ increases with concentration, but with a weaker dependence on $\phi$.

\footnotetext{
${ }^{28}$ Experiments that seem to support this prediction, although for an unknown value of $B_{2}$, are discussed in [99], where an interesting extensive analysis concerning upper bounds on $v(\Phi)$ is presented.
}

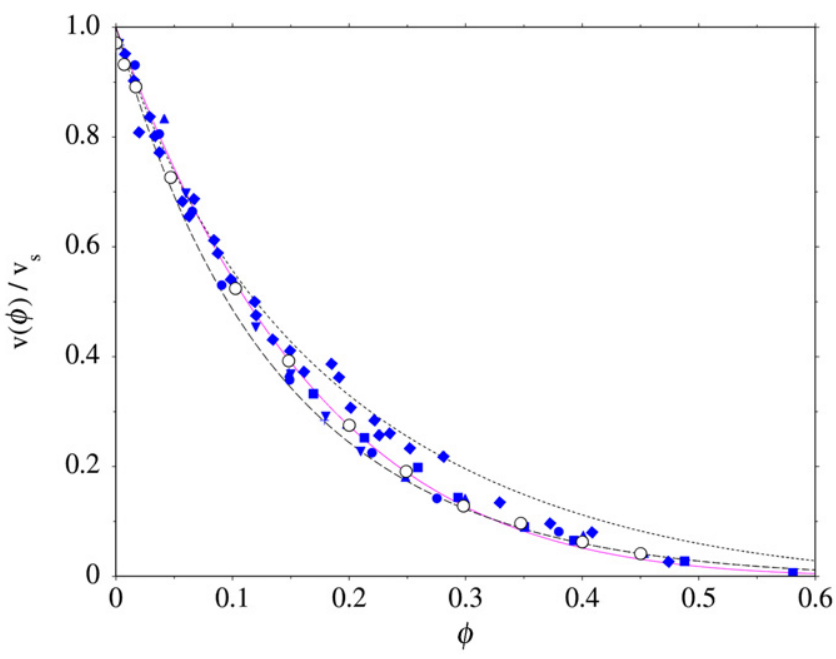

Figure 6. Experimental data for $v(\phi)$ from $[101](\bullet),[102](\boldsymbol{\Delta})$, [103] ( ), [104] ( $)$, and [105] ( $\mathbf{\nabla})$, together with theoretical results by Beenakker \& Mazur [95] (- - $)$ and Hayakawa \& Ichiki [96](- $--)$, and numerical simulations by Ladd [97] (O). The full line is a best fit with the empirical expression $v(\phi)=(1-\phi)^{\alpha}$, with $\alpha \simeq 5.8$.

\subsection{The quiet realm of macroscopic settling: time-invariant kinetic profiles}

If some delicate experimental issues are properly addressed (see 3.4), the sedimentation of a suspension of Brownian particles is a slow, regular collective process, which can be accurately described by a continuum equation. Neglecting the effects of fluctuations discussed in the next section, the expression for the mass flux in the presence of gravity leads, together with the continuity equation, to the following kinetic equation [87, 105]:

$$
\frac{\partial \phi}{\partial t}-v_{0} \frac{\partial[\phi H(\phi)]}{\partial z}=D_{0} \frac{\partial}{\partial z}\left\{H(\phi) \frac{\partial[\phi Z(\phi)]}{\partial \phi} \frac{\partial \phi}{\partial z}\right\},
$$


where $Z(\phi)=\Pi(\phi) / n k_{\mathrm{B}} T$ is the osmotic compressibility factor, and use has been made of equation (18). During most of the settling period, the dynamic sedimentation profiles obtained from equation (19) for an initially uniform suspension at volume fraction $\phi_{0}$ display a uniform column at $\phi=\phi_{0}$, merging above with the particle-devoid supernatant by a more or less expanded 'fan', and below with a dense sediment (the 'cake') that builds up from the cell bottom. Notably, equation (19) has a Burgers-like structure [106]; this means that the long-time asymptotic profile of the fan region behaves as a shock front moving with a constant speed and a time-invariant shape dictated by the competition between the $\phi$-dependent settling velocity, which sharpens the fan, and Brownian diffusion, which conversely spreads the settling front. This asymptotic behavior is reached on a time scale that depends on concentration, becoming very long at low $\phi^{29}$.

This result has some interesting features [105]. First of all, the top of the fan where $\phi \ll \phi_{0}$ has an exponential shape, like the dilute part of the equilibrium profile, which is however set by a different 'dynamic' sedimentation length $\ell_{\mathrm{g}}^{d y n}\left(\phi_{0}\right)=\ell_{\mathrm{g}}\left[1-H\left(\phi_{0}\right)\right]^{-1}$ which, if $H(\phi)$ decreases monotonically with $\phi$, is larger than $\ell_{\mathrm{g}}$. Moreover, the general solution for the shock-wave front yields the relation:

$$
\frac{H(\phi)}{H\left(\phi_{0}\right)}=\left[1+\ell_{\mathrm{g}} \frac{\partial[\phi Z(\phi)]}{\partial \phi} \frac{\partial \phi}{\partial z} \phi^{-1}\right]^{-1}
$$

hence, if $Z(\phi)$ is known from the EOS obtained at equilibrium, the whole behavior of $H(\phi)$ up to $\phi_{0}$ can be extracted from a single measurement of the kinetic settling profile $\phi(z)$ and of its derivative. Conversely, when $v(\phi)$ is known from standard settling measurements, equation (20) allows obtaining the equilibrium EOS up to $\phi_{0}$ from a single measurement of a kinetic settling profile, as sketched in figure 7.

It is worth recalling that the former analysis strictly applies only to the Brownian regime. Nevertheless, very promising extensions to higher Peclét numbers are feasible by making use of the so-called 'dynamic DFT' [107], in which an approximate deterministic equation for the temporal evolution of the average particle number density $n(\boldsymbol{r}, t)$ is derived from the stochastic Langevin equation by assuming that, out of equilibrium, the correlation function $\langle n(\boldsymbol{r}$, t) $\left.n\left(\boldsymbol{r}^{\prime}, t\right)\right\rangle$ has the same properties as in equilibrium. In particular, using the approximate expression for $H(\phi)$ given in [96], Royall et al [108] managed to obtain accurate kinetic sedimentation

profiles that are in close agreement up to values $P e \gtrsim 1$ with both numerical simulations and confocal microscopy studies of hard-sphere colloids confined in capillaries [108], and which reduce to those predicted by the 'batch settling' equation (19) for $P e \ll 1$. This approach proves then to be extremely useful in investigating layering structures that build up close to the walls at the particle length scale, or more complex effects such as the lateral patterns associated to the Rayleigh-Taylor instability which occurs when a cell containing a suspension at sedimentation equilibrium is turned upside down [108].

29 For dilute suspensions, using the Batchelor's result for $v(\phi)$ and neglecting the weak $\phi$-dependence of $D$, the time to reach steady-state is17 found to be approximately $10^{-2} D_{0} /\left(v_{s}^{2} \phi^{2}\right)$.

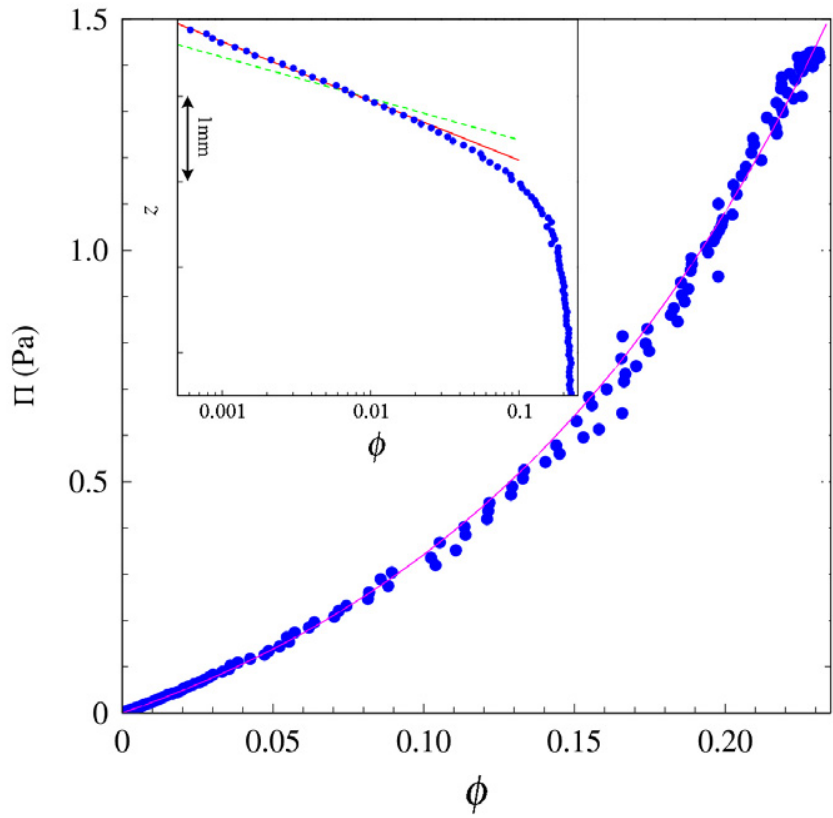

Figure 7. Comparison of the HS EOS (full line) with the results derived from the stationary settling profile of a MFA suspension at initial volume fraction $\phi_{0}=0.23$ shown in the inset, using the dependence of the settling velocity on $\phi$ obtained by tracking the time displacement of the meniscus [105]. The inset also shows that $\ell_{\mathrm{g}}^{d y n}$, given by the slope of the full line, is noticeably larger than $\ell_{\mathrm{g}}$ (dashed line).

\subsection{The frantic face of sedimentation: velocity fluctuations}

Unfortunately, Batchelor's clever renormalization scheme does not work for the mean square velocity fluctuations $\left\langle\delta v^{2}\right\rangle=\left\langle v^{2}\right\rangle-\langle v\rangle^{2}$, which are not quenched by the solvent backflow and, in fact, turn out to be unbounded. This surprising result was originally pointed out in a seminal paper by Caflisch and Luke [109], who showed that, summing up the perturbations due to the HI, $\left\langle\delta v^{2}\right\rangle \sim v_{s}^{2} \phi L / a$, where $L$ is the size of the container holding the suspension. Huge fluctuations, far larger than those due to thermal Brownian motion $\left(\left\langle\delta v^{2}\right\rangle_{\mathrm{th}}=k_{\mathrm{B}} T / m\right)$ are indeed observed in experiments, which, however, have been so far limited to suspensions of non-Brownian particles, where the evaluation of $\left\langle\delta v^{2}\right\rangle$ can be done by particle image velocimetry [110], direct tracking of tracer particles [111], or ultrasound correlation spectroscopy [112]. Apparently, however, these large- and long- ranged fluctuations do not diverge with the container size. Even more, intriguing experiments by Segrè $e t$ al [110] not only suggest that the velocity fluctuations are system-size independent, but also that the amplitude and the correlation length $\xi$ of the velocity fluctuations are universal in terms of

the particle volume fraction: $\left\langle\delta v^{2}\right\rangle^{1 / 2} \sim v_{s} \phi^{1 / 3} ; \xi \sim a \phi^{-1 / 3}$. This blatant contradiction with the theoretical predictions has given rise to a heated debate, which has been summarized in several excellent reviews [10,113-115]. For our purposes, it is sufficient to briefly discuss the main tentative explanations that have been proposed, in view of the corresponding situation for Brownian particles.

A key assumption of the Caflisch and Luke argument is that velocity fluctuations do not couple with concentration 
fluctuations: namely, they do not induce or modify structural correlations in the suspension. At high $P e$, however, this can be surely questioned. Koch and Shaqfeh [116], for instance, argued that HI might actually lead to a net mass deficit $\Delta m=$ $m^{*}$ (with respect to the equilibrium particle distribution) in a spherical volume $V$ with a radius comparable to $\xi$, surrounding a given test particle of buoyant mass $m^{*}$ : if this is the case, the overall volume $V$ turns out to be neutrally buoyant and does not show any more velocity fluctuations, which are therefore quenched beyond a length scale of order $\xi$. As worked out in full detail by Felderhof [117], who suggested that such a mechanism may be provided by a combination of a destructive interference of the individual particle flow patterns and of a rearrangement of particle positions,${ }^{30}$, this implies, for a dilute suspension, an anisotropic steady-state structure factor of the form:

$$
S(\boldsymbol{q})=\left[1-\mathrm{e}^{-q^{2} \xi^{2} \psi\left(\theta_{q}\right) / 4}\right]^{2},
$$

where $\psi\left(\theta_{\boldsymbol{q}}\right)$ is a generic angular function satisfying $\psi(\pi / 2)$ $=1, \psi(\pi-\theta)=\psi(\theta)$, which vanishes as $(q \xi)^{4}$ for $q \rightarrow 0$. One of the consequences of these non-equilibrium correlations is that, over length scales comparable to $\xi$, the spontaneous concentration fluctuations in the suspension should show significant suppression compared to the Poisson statistics expected for a dilute suspension and become anisotropic too, features which have apparently been observed in experiments $[118]^{31}$.

An alternative mechanism [114, 119] is based on the observation that, as originally pointed out by Lee et al [120], the presence of strong hydrodynamic velocity fluctuations on top of the Brownian ones may consistently modify the spreading of the sedimentation front. Indeed, HI yield an additional non-Brownian diffusive mechanism, which can be described by introducing a hydrodynamic 'dispersion' coefficient. Hydrodynamic dispersion is in general anisotropic, namely, a tensorial quantity: yet, if the velocity fluctuations are screened the way experiments suggest, a simple scaling argument shows that the dispersion coefficient should scale as $D_{h} \sim a v_{s}$ (although with a different amplitude along or perpendicularly to $g$ ), thus $D_{h} / D_{0} \sim P e$. Therefore, when $P e \gg 1$, hydrodynamic dispersion provides a much more efficient diffusion mechanism than Brownian diffusion: because of this, the sedimentation front spreads very fast, possibly without reaching its time-invariant shape, if $\phi$ is small, before settling is completed. Within the spreading fan, the suspension is then a 'stratified' fluid that quenches velocity fluctuations, because the downward-oriented concentration gradient limits how far down a heavy concentration fluctuation can fall, or how far up a light one can float: in other words, stratification acts as a stabilizing mechanism against fluctuations. As a matter of fact, experiments show that

\footnotetext{
30 The suggested destructive interference effect is very similar to what occurs for optical dipoles, leading to the Ewald-Oseen extinction theorem, whereas structural rearrangements resemble those occurring in the Debye-Hückel theory of electrolytes [117].

${ }^{31}$ It is also useful to point out that the theoretical results in [91], which are so nicely corroborated by the numerical simulations in [92], require us to assume the presence of the long-range Koch-Shaqfeh correlations, and in fact indirectly support the latter.
}

velocity fluctuations are much weaker in the fan than away from it, where $\mathrm{d} \phi / \mathrm{d} z$ is much smaller [121].

These two arguments, however, do not apply to Brownian particles: at low $P e$, indeed, $S(q)$ should not appreciably differ from the equilibrium structure factor and, provided that the initial volume fraction is not too small, the settling profile should rapidly reach the time-invariant profile discussed in 3.2 , characterized by a substantially extended 'column' where no stratification occurs. There is however a third mechanism that might in principle quench the velocity fluctuations even at low $P e$. The argument, originally suggested by Hinch [122] and then refined and supported by simulations by Ladd [123], is based on two considerations: (i) that velocity fluctuations are physically due to the presence of spontaneous number density fluctuations (Poisson-like, for a dilute system) yielding a spatially-varying buoyancy; (ii) that the presence of a bottom cell wall, however, unavoidably generates convection currents that conversely tend to homogenize the suspension: a fluctuation of size $\xi$ is predicted to convect with a velocity scaling as $v_{c}(\xi) \sim(\phi \xi /$ $a)^{1 / 2} v_{s}$, hence to be swept away on a time scale $t_{c} \sim(\xi /$ $a \phi)^{1 / 2} t_{s}$. The invoked mechanism is therefore roughly the following: the energy fed by gravity into macroscopic fluctuation is drained by a collective, albeit complex, convection pattern, being eventually dissipated at the cell bottom and at the interface between the suspension and the supernatant because of the no-slip boundary condition ${ }^{32}$. Fluctuations are then quenched, unless they build up on a time scale $t_{\xi}<t_{c}$. Since $t_{\xi} \sim \xi^{2} / D_{h} \sim \xi^{2} / v_{s} a$, the largest fluctuations will have a size $\xi_{\max } \sim a \phi^{-1 / 3}$, in agreement with experiments. When $D_{0} \gg D_{h}$, however, we should rather write $t_{\xi}=\xi^{2} / D_{0}$, yielding $\xi_{\max } \sim a \phi^{-1 / 3} P e^{-2 / 3}$, which still diverges for $P e \rightarrow 0$.

For Brownian particles, therefore, no mechanism that could invalidate the Caflisch \& Luke conclusions has to my knowledge been proposed. Unfortunately, the effect of a finite Peclét number on $\left\langle\delta v^{2}\right\rangle$ has been addressed only sporadically, with the noticeable exception of numerical investigations by Padding and Louis [90, 124]. This detailed study of the interplay between Brownian and hydrodynamic fluctuations contains several interesting results. For instance, $S(q)$ shows rather small deviations from equilibrium, which are confined to $q a \ll 1$, up to significantly high Peclét numbers

$(P e \gtrsim 10),{ }^{33}$ Moreover, the velocity fluctuations do seem to diverge with the container size, and are anisotropic: along

the direction of $g,\left\langle\delta v^{2}\right\rangle_{\|} \propto v_{s}^{2} \phi L / a$, whereas $\left\langle\delta v^{2}\right\rangle^{1 / 2} \simeq$ $\left\langle\delta v^{2}\right\rangle^{1 / 2} / 1.8$, in agreement the experimental findings [ 110$]$.

Hydrodynamic fluctuations are also clearly evidenced by the presence of a long-time tail in the velocity time-correlation function, which for $t \gg t_{s}$ decays exponentially with a time constant $\tau_{H} \sim(a / L)^{-1 / 2} \phi^{-1 / 2} t_{s}$ that diverges with the container size $L$. However, hydrodynamic fluctuations,

${ }^{32}$ Curiously, this mechanism is somehow the reverse of the energy cascade in turbulence: here energy is fed at the microscopic level and dissipated at the largest macroscopic length scale.

33 This basically supports Bachelor results for $v(\Phi)$ even when Brownian fluctuations are included, showing that the main contribution to the solvent back-flow is correctly evaluated using the equilibrium distribution around a test particle (see footnote on page 15), whereas the additional long-range contribution due to $\mathrm{HI}$ remains unchanged. 
although unbounded, may be hard to detect experimentally. A quantitative assessment of their relative strength with respect to thermal fluctuations yields indeed

$$
\frac{\left\langle\delta v^{2}\right\rangle_{h}}{\left\langle\delta v^{2}\right\rangle_{\text {th }}} \simeq \alpha \operatorname{Re} \operatorname{Pe} \phi \frac{L}{a},
$$

where the value of the prefactor $\alpha$ depends on whether fluctuation along or perpendicular to $g$ are considered, but in both cases it is of the order of $10^{-2}$. At low $P e$, and given the very small value of the Reynolds number in colloidal settling, this ratio will be quite small even for $L=10^{4}$ $-10^{5} a$. Unfortunately, the simulation box used in these numerical simulations is still too small to properly address the question of screening. On the experimental side, as we already mentioned, measurements have been so far limited to the non-Brownian regime, due to the lack of experimental methods for tracking the motion of particles that cannot be optically resolved. This experimental gap could be filled by exploiting novel optical correlation techniques that simultaneously yield the Brownian dynamics and the velocity field of sub-resolution particles [125, 126].

\subsection{Loose ends and booby traps in settling kinetics}

In my view, the puzzle of fluctuations has probably drained too much the efforts of the community dealing with sedimentation. There are indeed several baffling and practically important questions that surely deserve more attention. Just to give a few examples:

- The competition between settling and crystallization in HS suspensions has been addressed in the past, both in natural sedimentation [127] and, to some extent, in centrifugation [128]. These investigations have shown that the observed crystal growth is accounted for by the Wilson-Frenkel model, which relates the growth rate to the volume fraction of the metastable fluid in contact with the crystal interface. By increasing $P e$, and therefore the rate of accumulation at the cell bottom, crystal growth is eventually quenched (because crystals do not have enough time to incorporate the particles) and an amorphous glassy sediment forms. Yet, the detailed structure of the latter deserves further investigation. For instance, how does the compactness of the sediment depend on $P e$, or on the initial volume fraction of the suspension $\phi_{0}$ ? The latter issue has been investigated in a preliminary study, which qualitatively shows that denser suspensions form denser amorphous sediments [129], but a detailed analysis is still missing. In particular, it would be very interesting to investigate whether, under specific conditions, the concentration of the sediment close to the cell bottom can reach values close to RCP, hence generating a so-called 'hyper-uniform' structure with very unusual properties [130].

- Several natural settling phenomena (for instance, in the marine environment) take place in a fluid where density is inhomogeneous, namely, in a stratified solvent. An important question is whether settling in a fluid with a spatially variable density $\rho_{0}(z)$ is hydrodynamically stable, which of course means that the suspension density $\rho(z)$ must increase monotonically with depth $z(\mathrm{~d} \rho(z) /$ $\mathrm{d} z>0)$ during the whole settling process. Blanchette and Bush [131] have developed a general criterium for the occurrence of instabilities, which in particularly states that no hydrostatically unstable profile can develop due to particle settling if the fluid density gradient becomes more negative with increasing depth $\left(\mathrm{d}^{2} \rho_{0} / \mathrm{d} z^{2}\right.$ $>0$ ); simple but ingenious model experiments seem to fully support this result [131]. Given the practical importance of this issue, further studies aimed at investigating the nature of the instability patterns that build up if this condition is violated would be very useful.

- Even more puzzling is the question of the stability of settling processes in colloid mixtures. Batchelor has derived an elegant criterium for the hydrodynamic stability of a binary colloidal mixture, showing that settling becomes unstable when [93]

$$
\left[\frac{\partial\left(\phi_{1} v_{s 1}\right)}{\partial \phi_{1}}+\frac{\partial\left(\phi_{2} v_{s 2}\right)}{\partial \phi_{2}}\right]^{2}+4 \phi_{1} \phi_{2} \frac{\partial v_{s 1}}{\partial \phi_{2}} \frac{\partial v_{s 2}}{\partial \phi_{1}}<0 .
$$

Note that this requires, at least, that $\partial v_{s 1} / \partial \phi_{2}$ and $\partial v_{s 1} /$ $\partial \phi_{2}$ have opposite signs. If this condition holds, horizontal concentration gradients build up and lead to the formation of complex vertical columnar structures that are in fact observed in experiments [132]. This is often the case when the two species settle in opposite directions (one sediments and the other creams); it may also happen for two species which are both denser or lighter than the solvent, but only provided that the concentration of each species is quite high (say, larger than 15-20\%). However, we have recently observed complex instability patterns also developing in binary mixtures which are very diluted, with one of the two species present only in tracer amount [22]. These instabilities cannot be accounted for by Batchelor's theory, and probably involve, in a subtle way, hydrodynamic fluctuations.

Given the length this review has already reached, I have to refrain from expatiating on these issues, which, more than unsettled, are unsettling. Before ending this flying visit to the world of sedimentation dynamics, however, we had better add some notes of warning about experiments, which may actually run into several pitfalls.

First, gravity rigorously calls for an 'upright stance', namely, the cell where the experiment is performed must be as vertical as possible. Sedimentation in an inclined container is very different, because of the so-called 'Boycott effect' $[86,133]$, which may actually boycott any attempt to obtain reliable values for the settling velocity. If sedimentation takes place in a tilted cuvette, the particles tend indeed to settle close to the lower-lying lateral side, leaving close to the upper side a clear layer of solvent that can rise more easily, since it does not have to back-flow through the suspended particles; this may generate a convective roll that considerably speeds up settling ${ }^{34}$.

34 An interesting case where the Boycott effect is curiously quenched has been recently found in an interesting study [134] of sedimentation in confined geometries of very large particles: here confinement is found to increase the settling speed, but tilting the cell does not yield any further speed-up effect. 
Second, the settling cell must be wrapped up in a suitable 'thermal blanket', because sedimentation can be severely affected by temperature gradients; indeed, a quiescent fluid cannot stand any horizontal temperature gradients, even minimal, without convecting. Convective effects can be reduced if the cell is thin, which obviously limits lateral temperature differences $\Delta T$, and not too tall, so to reduce the convection velocity, but even for very short cells the latter may still be quite large compared to the typical settling speed. An efficient method to effectively quench convection is applying a weak stabilizing vertical temperature gradient, but this requires some caution. One would indeed naively think that a fluid is necessarily stable when heated from above, but for a colloidal suspension this may be wrong, because of particle thermophoresis, a cross-effect between heat and mass transport akin to the Soret effect in simple fluid mixtures [135]: if the particles have a negative Soret coefficient, namely, if they are driven by the thermal gradient to the hot, heating from above maybe the worst choice.

Finally, limited convection effects in sedimentation may actually be unavoidable due to boundary conditions. Indeed, close to the cell side walls there is a particle-depleted layer, which makes the solvent backflow non-uniform, generating a so-called 'intrinsic' convection effect which may be nonnegligible at low volume fraction [136].

\section{A final surprise: buoyancy in a crowded world}

The Archimedes' principle is arguably one of the oldest laws of physics; yet, it is worth pondering equation (1) for the buoyant mass, because there are specific situations where this concept does not seem to be so straightforward. Consider for instance an isolated particle 1 , of volume $v_{1}$ and density $\rho_{1}$, settling in a fluid which is itself a suspension of particles of type-2, differing in size and/or density from particle 1, at volume fraction $\phi_{2}$ : what density value should we use to evaluate $\Delta \rho$ in the expression for $\boldsymbol{F}_{b}$ ? This issue has been highly debated and a subject of many controversies; by analogy with the case of settling in a simple liquid mixture, most investigators took for granted that, instead of the density $\rho_{0}$ of the bare solvent, the density $\rho_{s}=\rho_{0}+\left(\rho_{2}-\rho_{0}\right) \phi_{2}$ of the suspension should be used [137, 138], although this assumption has been often criticized $[139,140]$. Let us restate the question as follows: can we replace the suspension of 'host' particles 2, which affects the settling of our 'guest' particle 1 via direct and HI, with an effective medium having a suitable density and viscosity?For what concerns the latter, the answer is definitely negative: condensing the complex effects of HI into a 'renormalized' viscosity does not work, unless the size ratio $r=a_{2} / a_{1}$ is very small. For density, as we shall see, a general answer does exist ${ }^{35}$.

35 Another experimental situation where 'anomalous' buoyancy effects are observed concerns density-gradient ultracentrifugation (DGU), a widely used analytical method that consists of adding to a biological solution a heavy but small species to generate a density gradient in the solvent by ultracentrifugation: this leads proteins, nucleic acids, or cellular organelles to accumulate in a thin band around the 'isopycnic point', namely, the position $z$ where the local solvent density is equal to the density of the species to be fractionated. For a discussion of DGU buoyancy anomalies see [141]
As a matter of fact, a correct thermodynamic analysis of sedimentation and centrifugation in multi-component fluids casts consistent doubts on the use of the simple Archimedes' expression for buoyancy. We already know (see footnote on page 2) that, even for a single species $i$ settling in a simple fluid, the buoyant mass should rather be written in terms of the specific volume $\overline{v_{i}}$, which can depend on nature of the solvent. In the presence of several other components $\{j\}$, even this correction is no longer sufficient, and the general thermodynamic expression for $m_{i}^{*}$ is given by [142]

$$
m_{i}^{*}=k_{\mathrm{B}} T\left(\frac{\partial \Pi}{\partial c_{i}}\right)^{-1}\left(\frac{\partial \rho_{s}}{\partial c_{i}}\right)_{\mu_{j}} \underset{c_{i} \rightarrow 0}{\longrightarrow} m_{i}\left(\frac{\partial \rho_{s}}{\partial c_{i}}\right)_{\mu_{j}}
$$

where $c_{i}$ is the mass concentration of component $i$, and the subscript $\mu_{j}$ indicates that the derivative has to be taken at constant chemical potential (not concentration) of all the other species. Unfortunately, this important warning has not been adequately considered by either colloid scientists nor by a large proportion of the biologists using centrifugation as a standard lab tool [143], arguably because the difference between equation (21) and the Archimedes' expression was not backed by any microscopic physical picture of buoyancy.

Rather surprisingly, a very general microscopic expression for buoyancy, which provides a firm microscopic basis to equation (21), can be obtained by a very simple mechanical equilibrium argument, which basically leads us to the conclusion that, in spite of any apparent discrepancies, the buoyancy force on a particle is nevertheless always given by the amount of 'displaced fluid', provided that the latter concept is correctly revisited and interpreted [144]. The key point of the argument is that, when the suspending fluid is a colloidal suspension, a solution containing other molecular species, or even a simple liquid showing sufficiently longranged spatial correlations, the amount of 'displaced' fluid is substantially modified by the density perturbation induced by the particle itself in the surroundings, which are quantified by the mutual radial distribution function $g_{12}(r)$. Consider indeed a region $\mathcal{V}$ in the fluid large enough to include the whole perturbed region, for instance a sphere with a radius $R$ much greater than the range of $g_{12}(r)$. Prior to particle insertion, the pressure forces from the outer fluid must balance, at equilibrium, the total weight of the 'fluid' (which in the present context we regard, for the sake of generality, as a nondescript 'component 2') in $\mathcal{V}, \boldsymbol{W}=-m_{2} n_{2} g \mathcal{V} \hat{z}$, where $\hat{z}$ is the unit vector along the vertical. After the particle has been inserted, the weight in $\mathcal{V}$ becomes $\boldsymbol{W}^{\prime}=-m \int_{2} g n_{2}$ $\mathrm{d}^{3} r g_{12}(r) \hat{z}$, and $\boldsymbol{F}_{b}$ is simply

given by the unbalanced mechanical force due to the total mass change in $\mathcal{V}$ :

$$
\boldsymbol{F}_{b}=\boldsymbol{W}^{\prime}-\boldsymbol{W}=-4 \pi m_{2} g n_{2} \int_{0}^{\infty} \mathrm{d} r r^{2}\left[g_{12}(r)-1\right] \hat{\boldsymbol{z}},
$$

where the integral has been extended to the whole space because $g_{12}(r) \equiv 1$ outside $\mathcal{V}$. Using a plain but effective image, this 'generalized Archimedes principle' (GAP) states that a settling particle unavoidably carries around with itself the 'dressing' made of the surrounding correlated region, which modifies its effective weight. Although obtained with 
a simple mechanical argument, the GAP is valid regardless of the specific nature of the surrounding 'fluid', with the only basic assumption that the guest particles are so diluted that self-interactions between them can be neglected. When the surrounding fluid is a dispersion in a solvent of density $\rho_{0}$ of particles of volume $v_{2}$, made of a material with density $\rho_{2}$, equation (22) is still valid, provided that $m_{2}$ is substituted by $m_{2}^{\prime}=\left(\rho_{2}-\rho_{0}\right) v_{2}$, and that $\boldsymbol{F}_{b}$ is taken as the excess buoyancy adding to the buoyant force $\rho_{0} v_{1} g \hat{z}$ due to the bare solvent. Whereas for a molecular solvent corrections to the standard Archimedes' expression are small unless the guest particle is nanometric, here the buoyancy can be widely different, leading to unexpected and counterintuitive effect.

This general result, which can also be rigorously obtained by DFT [145], can be given a thermodynamic interpretation by means of the Kirkwood-Buff theory of dilute solutions [141], yielding in the dilute limit the expression (21) for $m^{*}$, and for the amplitude of buoyancy force

$$
F_{b}=m_{2}^{\prime} g\left[\frac{\partial \Pi}{\partial n_{1}}-k T\right]\left[\frac{\partial \Pi}{\partial n_{2}}\right]^{-1},
$$

where $m_{2}^{\prime}=\left(\rho_{2}-\rho_{0}\right) v_{2}$ is the 'Archimedean' buoyant masses of the host species. Equation (23) lends itself to a clear physical interpretation: while the first factor depends on the specific interactions between the two species, the second one shows that $F_{b}$ is related to response of the host fluid via the osmotic compressibility of the suspension.

The consistency of the GAP can be checked by considering the apparently dissimilar case of a system made of just one kind of colloidal particles interacting via arbitrary forces and not necessarily dilute, where we already know that the sedimentation profile is obtained from equation (6). Nevertheless, we can ideally tag as 'component 1' a single particle, regard it as an isolated 'guest' settling in a sea of identical particles, and evaluate the profile using equation (23) where components 1 and 2 are identified. A simple argument shows that this alternative approach is fully equivalent to the former [141]. Therefore, to evaluate the sedimentation profile of a single-component concentrated suspension we can either regard it as a system of interacting particles and use equation (6), or find the equilibrium probability distribution for a single particle, subjected however to the buoyancy exerted by an effective medium of density

$$
\rho^{*}\left(n_{1}\right)=\rho_{0}+\left[1-k_{\mathrm{B}} T\left(\frac{\partial \Pi}{\partial n_{1}}\right)^{-1}\right]\left(\rho_{1}-\rho_{0}\right) .
$$

These theoretical ideas have been experimentally tested by considering the equilibrium sedimentation profile of model colloidal mixtures [144]. Consider the simple case of a tiny amount of spherical particles with radius $a_{1}$ and density $\rho_{1}$ dispersed in a generic colloidal fluid of HS of radius $a_{2}=$ $q a_{1}$, with $q \ll 1$, made of a material of density $\rho_{2}$. Unless the host suspension is very concentrated, the largest contribution to the integral in equation (22) comes from the region $a_{1}<$ $r<a_{1}+a_{2}$. Hence, we can take for $g_{12}(r)$ its limiting value for $\phi_{2} \rightarrow 0$

$$
g_{12}(r) \simeq\left\{\begin{array}{cc}
0 & \text { for } r \leqslant a_{1}+a_{2} \\
1 & \text { otherwise }
\end{array}\right.
$$

which amounts to state that the excess buoyancy comes only from the (buoyant) mass of type-2 particles excluded from the 'depletion' region $r<a_{1}+a_{2}$. Thus we obtain

$$
\boldsymbol{F}_{b}=\frac{4 \pi}{3}\left(a_{1}+a_{2}\right)^{3} n_{2} m_{2}^{\prime} g \hat{z}=\left(\rho_{2}-\rho_{0}\right)(1+q)^{3} v_{1} g \phi_{2} \hat{z}
$$

where $\phi_{2}=v_{2} n_{2}$ is the volume fraction of type- 2 particles. The host suspension acts therefore on the guest particle with a total upward thrust $\boldsymbol{F}_{b}+\rho_{0} v_{1} g \hat{\boldsymbol{z}}$, which amounts to the buoyant force exerted by a homogeneous solvent of density $\rho^{*}=\rho_{0}+(1+q)^{3}\left(\rho_{2}-\rho_{0}\right) \phi_{2}$, which, when $\rho_{2}>\rho_{0}$, is always larger than the density of the host suspension $\rho_{s}$. A straightforward consequence is that the weight of a type-1 particle is exactly balanced by a suspension of type- 2 particles at a volume fraction

$$
\phi_{2}^{*}=\frac{\phi_{2}^{\text {iso }}}{(1+q)^{3}}
$$

which, even for a size ratio as small as $q=0.1$, is about $30 \%$ lower than the 'isopycnic' value $\phi_{2}^{\text {iso }}=\left(\rho_{1}-\rho_{0}\right) /\left(\rho_{2}-\rho_{0}\right)$ one would get by assuming $\rho^{*}=\rho_{s}$. Measurements of the equilibrium distribution profile of a tiny amount of poly(methyl methacrylate) particles (PMMA), with a size $a_{1}$ ranging between 200 and $500 \mathrm{~nm}$, dispersed in host suspensions of MFA particles of radius $a_{2}=90 \mathrm{~nm}$, yield effective isopycnic points that are in excellent agreement with the theoretical predictions of the GAP.

When the volume fraction $\phi_{2}$ of the host particles is not small, or for a generic size ratio $q$, this simple excluded volume approximation cannot be used any longer. An explicit evaluation of the buoyancy force may nevertheless be obtained using the Mansoori-Carnahan-Starling EOS for hard-sphere mixtures [146]. The results of a DFT analysis display a remarkable feature: whereas in the excluded volume approximation the effective density is predicted to increase linearly with $\phi_{2}$, for $q>1$ (namely, for a small particle settling in a sea of larger ones) $\rho^{*}$, and therefore the excess buoyancy force $F_{b}=-\left(\rho^{*}-\rho_{0}\right) v_{1} g$, attains a maximum for a value of $\phi_{2}$ that is substantially smaller than one. When $q \gg$ 1 , this maximum is attained for $\phi_{2} \simeq 0.155$ and is worth $F_{b} \simeq$ $0.055 m_{2} g$. Hence, the buoyancy force on a very small particle does not depend on the size of the particle itself, but only on the mass of the host particles, reaching an optimal value equal to the $5.5 \%$ of the gravitational force acting on them; remarkably, $F_{b}$ can then be much larger than the weight of the guest particle. These rather surprising predictions have been tested by dispersing in MFA suspensions a tiny amount of very dense gold nanoparticles $\left(\rho_{1}=19.3 \mathrm{~g} \mathrm{~cm}^{-3}\right)$ : at equilibrium, the gold particles were found to float mostly in the upper, very dilute region of the MFA equilibrium profile, where the local density of the solvent is as low as $\rho_{s} \simeq 1.2 \mathrm{~g} \mathrm{~cm}^{-3}$.

The applications of equation (22) are not however limited to HS mixtures. In the presence of a strong attractive contribution to the mutual interaction potential between the guest and host particles, for instance, the total buoyancy force on the particle can be reduced with respect to the Archimedes' value, because attractive forces yield a positive 
contribution to the integral of $h_{12}(r)$ for $r>a_{1}+a_{2}$ that can well overcompensate the negative excluded volume contribution, increasing its buoyant mass and yielding a kind of 'reversed' buoyancy. Physical systems where these predictions may be realized are binary mixtures of spheres with strong charge and size asymmetry, giving rise to the stabilizing 'nanoparticle halos' discussed for instance in [147, 148]. Even more instructive is considering what happens when the guest particles are not spherical, for instance if they are thin platelets or long rods. If the guest particle is a thin disk of radius $R$ and thickness $r \ll R$, settling in a 'sea' of spherical particles of radius $a_{2}$, in the excluded volume approximation the 'effective' isopycnic volume fraction is found to be $\phi_{2}^{*}=\phi_{2}^{\text {iso }} /\left(1+2 q_{r}\right)$, where $q_{r}=a_{2} / r$. Note that, even if the size of the host particles is much smaller than the disk radius $\left(a_{2} / R \ll 1\right)$, the excluded volume is $v^{e x} \simeq\left(1+2 q_{r}\right) v_{1}$, which, for a thin platelet, is much larger than $v_{1}$. In the case of a rod of length $L$ and radius $r$, a similar calculation yields $\phi_{2}^{*}=\phi_{2}^{\text {iso }} /\left(1+q_{r}\right)^{2}$. In both cases, buoyancy corrections are large when the ratio of the host particle size to the smallest dimension of the guest particle is large. When $q_{r} \gg 1, \phi_{2}^{*} / \phi_{2}^{\text {iso }}$ scales with $r / a_{2}$ for platelets, and with the same ratio squared for rods.

All the examples we have discussed refer to a guest particle surrounded by a suspension of colloidal particles of a different kind. However, equation (22) has been derived in the more general context of a particle suspended in a generic correlated fluid, of which a colloidal suspension is just an example. Even a simple solvent displays density correlations around the particle, although on the length scale of molecular interactions. For a particle the size of a few nanometers, such as a protein or a surfactant micelle, these density correlation effects may nonetheless be non-negligible, and provide, as a matter of fact, a microscopic physical meaning for the thermodynamic concept of specific volume. Much larger corrections to the buoyancy are expected when the solvent displays long-range density correlations, being for instance close to its critical point. In this case, depending on preferential wetting of the guest particle surface, a consistent interfacial layer with vapor-like or liquid-like density would indeed progressively grow around the particle surface, strongly modifying the particle buoyant mass. Experiments confirming this striking prediction could however be more easily performed in the conceptually equivalent situation of a particle dispersed in a critical liquid mixture.

It is worth recalling that the model we have described totally neglects self-interactions of the guest species, namely, the GAP properly describes only the sedimentation of very dilute tracers. A situation where self-interactions are surely not negligible is highlighted by recent experiments where heavier gibbsite platelets are found to float at equilibrium on top of a lighter silica sphere suspension [149]. Because they are rather concentrated, however, the platelets organize into a nematic liquid crystal phase: determining the equilibrium sedimentation profiles of the two species requires in this case a full DFT study of the phase diagram in the presence of gravity. It is however interesting to note that the experiments in [149] were actually motivated by a former DFT analysis of the equilibrium density profiles of colloid + polymer mixtures where the two species are assumed to have the same size ( $q=$ 1 ), but the polymer mass $m_{2}$ is assumed to be noticeably smaller than the mass $m_{1}$ of the colloidal particles [150]. Rather surprisingly, a colloidal liquid is found to float on top of a polymer solution which contains a negligible amount of particles. Once again, colloid-colloid interactions cannot be neglected, hence no accurate evaluation can be obtained from the GAP; yet, a simple 'dressed particle' picture allows us to qualitatively grasp such a non-intuitive effect, at least when the polymer solution is assumed to be ideal (namely, polymer-polymer interactions are fully neglected). Indeed, whereas the colloidal particles have to displace both the polymer and their like, a polymer coil needs to displace only the particles. Hence, the excess buoyancy contribution can be far larger for the particles than for the coils. It is instructive to see what we would expect in a condition where the GAP can be applied, namely, when the colloidal particles are very dilute. Because the polymer is ideal, its equilibrium profile is strictly barometric. Moreover, when self-interactions of the host species are neglected, the excluded volume approximation is actually exact, because correlations cannot extend beyond the excluded volume. Then, for the specific case $m_{2} / m_{1}=0.235$ considered in [150], the effective isopycnic point is readily

found to be $\phi_{2}^{*} \simeq 0.53$, which is comparable with the polymer volume fraction at the position corresponding to the lower boundary of the 'floating' colloid in figure 1 of [150].

Finally, it is important to point out that care must be taken in applying effective buoyancy concepts to settling dynamics, for equation (22) requires the structure of the surrounding fluid to be the equilibrium one. To derive the effective buoyancy at high Peclét number, theoretical investigations like those developed in [91] could be very useful.

\section{Conclusions: Die Entdeckung der Langsamkeit}

The reader who laboriously managed to reach this point, after wandering through so many settled, unsettled, and sometimes truly unsettling issues in sedimentation, may justly feel too exhausted to be snowed under by long concluding remarks. So, let us stick to the main messages I hope to have conveyed. Equilibrium sedimentation measurements can be a great tool for investigating fundamental problems in statistical mechanics. Sometimes, serendipitous discoveries such as the inflated profiles of charged colloids force us to reflect upon the very pillars on which colloid science stands. Although ingenious experimental solutions and new theoretical approaches have recently allowed us to survey a much wider class of soft matter systems, ample room still exists for further exciting investigations using centrifuges, whose power has surely not been fully exploited. The microscopic settling dynamics is plagued by multi-body hydrodynamic interactions that, like wicked trolls, generate gigantic velocity fluctuations whose properties are still partly understood. Nevertheless, such a deeply hidden mayhem leads to macroscopic kinetic settling profiles that bear additional information on the static and dynamic properties of a Brownian suspension. Several other baffling questions about settling kinetics, summarized in section 3.4 from a personal and 
arguably too narrow perspective, surely call for further theoretical and experimental efforts. As a matter of fact, reflecting upon the problem of buoyancy has led us to realize that even simple concepts in sedimentation may not be so trivial.

But there is a last, more important lesson that sedimentation teaches us: to perform sound investigations, we must arm ourselves with patience. Inflated profiles for charge colloids became manifest just because samples were left undisturbed for years. Even more, Laponite suspensions show that what we regard as an equilibrium state can be a delusion, and that drastic changes may take place after an exceptionally long time. As the great biographical novel by Sten Nadolny giving the title to this section tells us, slowness can often be a powerful asset, if combined with persistence. After all, it is on the strength of its persistent slowness that sedimentation created those majestic deposition landforms which contribute to making this planet a better place to live. Hoping, of course, that our frantic species does not spoil its labor.

\section{Acknowledgments}

During the past few years, and while preparing this review, I have enjoyed and profited from discussions about sedimentation with so many colleagues and friends that it would be silly to try and draw up a complete list, lest I forget any of them. I am also particularly grateful to those of them who granted me permission to reprint their original data and figures, and to P Cicuta, L Cipelletti, A Louis, A Parola, A Philipse, and A van Blaaderen for their careful reading and comments. Finally, let me address a very special acknowledgement to my co-worker Stefano Buzzaccaro, not only for the important contributions he has made to all the sedimentation studies made in my group, but also for the contagious enthusiasm he has always shown toward this exciting field of investigation. This work has been supported by PRIN 2012 funding from the Italian Ministry of Education and Research (MIUR).

\section{References}

[1] Julien P Y 2010 Erosion and Sedimentation (Cambridge: Cambridge University Press)

[2] Hind W C 1999 Aerosol Technology: Properties, Behavior, and Measurement of Airborne Particles 2nd edn (New York: Wiley-Blackwell)

[3] Kiørboe T 2008 A Mechanistic Approach to Plankton Ecology (Princeton, NJ: Princeton University Press)

[4] Woon-Fong Leung W 1998 Industrial Centrifugation Technology (New York: McGraw-Hill)

[5] Lebowitz J, Lewis M S and Schuck P 2002 Modern analytical ultracentrifugation in protein science-a tutorial review Protein Sci. 11 2067-79

[6] Perrin J 1909 Mouvement brownien et réalité moléculaire Ann. Chim. Phys. 18 5-114 Quoted is the translation made by Frederick Soddy in 1910 under the imprint of Taylor \& Francis, London, reprinted in 2005 by Dover Publications

[7] Ramsden W 1903 Separation of solids in the surface-layers of solutions and 'suspensions' Proc. R. Soc. Lond. 72 156-64

[8] Pickering S U 1907 Emulsions J. Chem. Soc. 91 2001-21

[9] Royall C P, van Roij R and van Blaaderen A 2005 Extended sedimentation profiles in charged colloids: the gravitational length, entropy, and electrostatics $J$. Phys.: Condens. Matter 17 2315-26

[10] Ramaswamy S 2001 Issues in the statistical mechanics of steady sedimentation Adv. Phys. 50 297-347

[11] El Masri D, Vissers T, Badaire S, Stiefelhagen J C P, Vutukuri H R, Helfferich P, Zhang T H, Kegel W K, Imhof A and van Blaaderen A 2012 A qualitative confocal microscopy study on a range of colloidal processes by simulating microgravity conditions through slow rotations Soft Matter 8 6979-90

[12] McMillan W G and Mayer J E 1945 The statistical thermodynamics of multicomponent systems $J$. Chem Phys. 13 276-305

[13] Dijkstra M, van Roij R and Evans R 2000 Effective interactions, structure, and isothermal compressibility of colloidal suspensions J. Chem. Phys. 1134799

[14] Biben T, Hansen J-P and Barrat J-L 1993 Density profiles of concentrated colloidal suspensions in sedimentation equilibrium $J$. Chem. Phys. 98 7330-44

[15] Nelson P C 2004 Biological Physics (New York: Freeman)

[16] Hachisu S and Takano K 1982 Pressure of disorder to order transition in monodisperse latex $A d v$. Colloid Interface Sci. $16233-52$

[17] Aarts D G A L, Schmidt M and Lekkerkerker H N W

2004 Direct visual observation of thermal capillary waves Science 304 847-50

[18] van der Linden M N, El Masri D, Dijkstra M and van Blaaderen A 2013 Expansion of charged colloids after centrifugation: formation and crystallisation of long-range repulsive glasses Soft Matter 911618

[19] Rutgers M A, Dunsmuir J H, Xue J-Z, Russel W B and Chaikin P M 1996 Measurement of the hard-sphere equation of state using screened charged polystyrene colloids Phys. Rev. B 53 5043-6

[20] Luigjes B, Thies-Weesie D M E, Philipse A P and Erné B H 2012 Sedimentation equilibria of ferrofluids: I. Analytical centrifugation in ultrathin glass capillaries $J$. Phys.: Condens. Matter 24245103

[21] Degiorgio V, Piazza R, Bellini T and Visca M 1994 Static and dynamic light scattering study of fluorinated polymer colloids with a crystalline internal structure $A d v$. Colloid Interface Sci. 48 61-91

[22] Piazza R, Buzzaccaro S and Secchi E 2012 The unbearable heaviness of colloids: facts, surprises, and puzzles in sedimentation J. Phys: Condens. Matt. 24284109

[23] Hansen J-P and McDonald I R 2013 Theory of Simple Liquids: with Applications to Soft Matter 4th edn (New York: Academic)

[24] Pusey P N and van Megen W 1986 Phase-behavior of concentrated suspensions of nearly hard colloidal spheres Nature 320 340-2

[25] Piazza R, Bellini T and Degiorgio V 1993 Equilibrium sedimentation profiles of screened charged colloids: a test of the hard-sphere equation of state Phys. Rev. Lett. 71 4267-70

[26] Carnahan N F and Starling K E 1969 Equation of state for nonattracting rigid spheres J. Chem. Phys. 51 635-6

[27] Daanoun A, Tejero C F and Baus M 1994 van der Waals theory for solids Phys. Rev. E 50 2913-24

[28] Buzzaccaro S, Rusconi R and Piazza R 2007 'Sticky' hard spheres: equation of state, phase diagram, and metastable gels Phys. Rev. Lett. 99098301

[29] Hagen M H J and Frenkel D 1995 Determination of phase diagrams for the hard-core attractive Yukawa system

$$
\text { J. Chem. Phys. } 101 \text { 4093-7 }
$$

[30] Bolhuis P and Frenkel D 1994 Prediction of an expandedto-condensed transition in colloidal crystals

Phys. Rev. Lett. 72 2211-4 
[31] Piazza R 2000 Interactions and phase transitions in protein solutions Curr. Opin. Colloid Interface Sci. 5 38-43

[32] Zaccarelli E 2007 Colloidal gels: equilibrium and non-equilibrium routes J. Phys.: Condens. Matter 19323101

[33] Lekkerkerker H N W and Tuinier R 2011 Colloids and the Depletion Interaction (Dordrecht: Springer)

[34] Stell G 1991 Sticky spheres and related systems J. Stat. Phys. 63 1203-21

[35] Baxter R 1968 Percus-Yevick equation for hard spheres with surface adhesion J. Chem. Phys. 49 2770-4

[36] Marenduzzo D, Finan K and Cook P R 2006 The depletion attraction: an underappreciated force driving cellular organization J. Cell Biol. 175 681-6

[37] Miller M A and Frenkel D 2004 Phase diagram of the adhesive hard sphere fluid J. Chem. Phys. $121535-45$

[38] Foffi G et al 2002 Phase equilibria and glass transition in colloidal systems with short-ranged attractive interactions: application to protein crystallization Phys. Rev. E 65031407

[39] Bolhuis P 1996 Liquid-like behaviour in solids, Solid-like behaviour in liquids $P h D$ Thesis Utrecht

[40] ten Wolde R P and Frenkel D 1997 Enhancement of protein crystal nucleation by critical density fluctuations Science 277 1975-7

[41] Lu P J, Zaccarelli E, Ciulla F, Schofield A B, Sciortino F and Weitz D A 2008 Gelation of particles with short-range attraction Nature $\mathbf{4 5 3} 499$

[42] Buzzaccaro S, Piazza R, Colombo J and Parola A 2010 Enhancement of depletion forces by electrostatic depletant repulsion J. Chem. Phys. 132124902

[43] Schmidt M, Dijkstra M and Hansen J-P 2004 Competition between sedimentation and phase coexistence of colloidal dispersions under gravity J. Phys.: Condens. Matter 16 S4185-94

[44] Biben T and Hansen J-P 1994 Sedimentation equilibrium in concentrated charge-stabilized colloidal suspensions $J$. Phys.: Condens. Matter 6 A345-9

[45] Philipse A P and Koenderink G H 2003 Sedimentation-diffusion profiles and layered sedimentation of charged colloids at low ionic strength Adv. Colloid Interface Sci. 100 613-39

[46] Donnan F G 1924 The Theory of Membrane Equilibria Chem. Rev. 173-90

[47] Philipse A and Vrij A 2011 The Donnan equilibrium: I. On the thermodynamic foundation of the Donnan equation of state J. Phys.: Condens. Matter 23194106

[48] van Roij R 2003 Defying gravity with entropy and electrostatics: sedimentation of charged colloids $J$. Phys.: Condens. Matter 15 S3569-80

[49] Raşa M and Philipse A P 2004 Evidence for a macroscopic electric field in the sedimentation profiles of charged colloids Nature 429 857-60

[50] Raşa M, Erné B H, Zoetekouw B, van Roij R and Philipse A P 2005 Macroscopic electric field and osmotic pressure in ultracentrifugal sedimentation-diffusion equilibria of charged colloids J. Phys.: Condens. Matter 17 2293-314

[51] Belloni L 2005 Yes, pair correlations alone do determine sedimentation profiles of highly charged colloids J. Chem. Phys. 123204705

[52] Torres A, Cuetos A, Dijkstra M and van Roij R 2007 Sedimentation of charged colloids: the primitive model and the effective one-component approach Phys. Rev. E 75041405

[53] Torres A, Cuetos A, Dijkstra M and van Roij R 2008 Breakdown of the Yukawa model in de-ionized colloidal suspensions Phys. Rev. E 77031402
[54] Luigjes B, Thies-Weesie D M E, Erné B H and Philipse A P 2012 Sedimentation equilibria of ferrofluids: II. Experimental osmotic equations of state of magnetite colloids J. Phys.: Condens. Matter 24245104

[55] Camp P J and Patey G N 2001 Coexistence and criticality of fluids with long-range potentials J. Chem. Phys. 114 399-408

[56] Vliegenthart G A and Lekkerkerker H N W 2000 Predicting the gas-liquid critical point from the second virial coefficient J. Chem. Phys. 112 5364-9

[57] Li N, Newman H D, Valera M, Saika-Voivod I and Yethiraj A 2010 Colloids with a tunable dipolar interaction: equations of state and order parameters via confocal microscopy Soft Matter 6 876-80

[58] Allen R, Goulding D and Hansen J-P 1999 Sedimentation equilibria of colloidal hard rod dispersions Phys. Chem. Commun. 2 30-3

[59] Savenko S V and Dijkstra M 2004 Sedimentation and multiphase equilibria in suspensions of colloidal hard rods Phys. Rev. E 70051401

[60] Kuijk A, Byelov D V, Petukhov A V, van Blaaderen A and Imhof A 2012 Phase behavior of colloidal silica rods Faraday Discuss. 159 181-99

[61] Bolhuis P G and Frenkel D 1997 Tracing the phase boundaries of hard spherocylinders J. Chem. Phys. 106 666-87

[62] Baulin V A and Khoklov A R 1999 Nematic ordering of rigid rods in a gravitational field Phys. Rev. E $602973-7$

[63] Wijnhoven J E G J, van't Zand D D, van der Beek D and Lekkerkerker H N W 2005 Sedimentation and phase transitions of colloidal gibbsite platelets Langmuir 21 10422-7

[64] Ruzicka B, Zaccarelli E, Zulian L, Angelini R, Sztucki M, Moussaid A, Narayanan T and Sciortino F 2011 Observation of empty liquids and equilibrium gels in a colloidal clay Nature Mater. 10 56-60

[65] Bianchi E, Largo J, Tartaglia P, Zaccarelli E and Sciortino F 2006 Phase diagram of patchy colloids: towards empty liquids Phys. Rev. Lett. 97168301

[66] Brambilla G, Buzzaccaro S, Piazza R, Berthier L and Cipelletti L 2011 Highly nonlinear dynamics in a slowly sedimenting colloidal gel Phys. Rev. Lett. 106118302

[67] Buzzaccaro S, Secchi E, Brambilla G, Piazza R and Cipelletti L 2012 Equilibrium concentration profiles and sedimentation kinetics of colloidal gels under gravitational stress J. Phys: Condens. Matter 24284103

[68] Seto R, Botet R, Meireles M, Auernhammer G K and Cabane B 2013 Compressive consolidation of strongly aggregated particle gels J. Rheol. 57 1347-66

[69] Princen H M 1986 Osmotic Pressure of foams and highly concentrated emulsions: I. Theoretical considerations Langmuir 2 519-24

[70] Mason T G, Lacasse M D, Grest G S, Levine D, Bibette J and Weitz D A 1997 Osmotic pressure and viscoelastic shear moduli of concentrated emulsions Phys. Rev. E $563150-66$

[71] Höhler R, Sang Y Y C, Lorenceau E and Cohen-Addad S 2008 Osmotic pressure and structures of monodisperse ordered foam Langmuir 24 418-25

[72] Maestro A, Drenckhan W, Rio E and Höhler R 2013 Liquid dispersions under gravity: volume fraction profile and osmotic pressure Soft Matter 9 2531-40

[73] Maurice L, Maguire R A, Schofield A B, Cates M E, Clegg P S and Thijssen J H J 2013 Squeezing particle-stabilized emulsions into biliquid foams - equation of state Soft Matter 9 7757-65

[74] Palacci J, Cottin-Bizonne C, Ybert C and Bocquet L 2010 Sedimentation and effective temperature of active colloidal suspensions Phys. Rev. Lett. 105088304 
[75] Enculescu M and Stark H 2011 Active colloidal suspensions exhibit polar order under gravity Phys. Rev. Lett. 107058301

[76] Cates M E 2012 Diffusive transport without detailed balance: does microbiology needs statistical physics? Rep. Prog. Phys. 75042601

[77] Nash R W, Adhikari R, Tailleur J and Cates M E 2010 Run-and-Tumble Particles with Hydrodynamics: Sedimentation, Trapping, and Upstream Swimming Phys. Rev. Lett. 104258101

[78] Barrett-Freeman C, Evans M R, Marenduzzo D and Poon W C K 2008 Nonequilibrium phase transition in the sedimentation of reproducing particles Phys. Rev. Lett. 101100602

[79] Rivas G and Minton A P 2011 Beyond the second virial coefficient: sedimentation equilibrium in highly non-ideal solutions Methods 54 167-74

[80] Rivas G, Fernandez J A and Minton A P 1999 Direct observation of the self-association of dilute proteins in the presence of inert macromolecules at high concentration via tracer sedimentation equilibrium: theory, experiment, and biological significance Biochemistry 38 9379-88

[81] dos Santos A P and Levin Y 2011 Ion specificity and the theory of stability of colloidal suspensions Phys. Rev. Lett. 106167801

[82] Duyndam A and Odijk T 1993 Equilibrium distribution of linear micellar aggregates in a gravitational or centrifugal field Langmuir 9 1960-1

[83] Papaseit C, Pochon N and Tabony J 2000 Microtubule self-organization is gravity-dependent Proc. Natl Acad. Sci. USA $978364-8$

[84] Baulin V A 2003 Self-assembled aggregates in the gravitational field: growth and nematic order J. Chem. Phys. 119 2874-85

[85] Cates M E and Candau S J 1990 Statics and dynamics of worm-like surfactant micelles J. Phys.: Condens. Matter 2 6869-92

[86] Nagele G 2013 Colloidal hydrodynamics Physics of Complex Colloids (Proc. Int. School of Physics 'Enrico Fermi', Course 184) ed C Bechinger, F Sciortino and P Ziherl (Amsterdam: IOS Press) pp 507-601

[87] Russel W B, Saville D A and Schowalter W R 1992 Colloidal Dispersions (Cambridge: Cambridge University Press) chapter 12

[88] Smoluchowski M S 1912 On the practical applicability of Stokes' law of resistance, and the modifications of it required in certain cases Proc. 5th Intern. Cong. Math.(Cambridge, UK) vol 2 192-201

[89] Batchelor G K 1972 Sedimentation in a dilute dispersion of spheres J. Fluid Mech. 52 245-68

[90] Padding J T and Louis A A 2008 Interplay between hydrodynamic and Brownian fluctuations in sedimenting colloidal suspensions Phys. Rev. E 77011402

[91] Cichocki B and Sadlej K 2005 Steady-state particle distribution of a dilute sedimenting suspension Europhys. Lett. 72 936-42

[92] Abade G C and Cichocki B 2012 Sedimentation of non-Brownian suspensions J. Phys.: Conf. Ser. 392012002

[93] Batchelor G K 1982 Sedimentation in a dilute polydisperse system of interacting spheres: I. General theory J. Fluid Mech. 119 379-408

[94] Thies-Weesie D M E, Philipse A P, Nagele G, Mandl B and Klein R 1995 Nonanalytical concentration dependence of sedimentation of charged silica spheres in an organic solvent: experiments and calculations $J$. Colloid Interface Sci. $17643-54$

[95] Beenakker C W J and Mazur P 1984 Diffusion of spheres in a concentrated suspension II Physica A 126 349-70
[96] Hayakawa H and Ichiki K 1995 Statistical theory of sedimentation of disordered suspensions Phys. Rev. E 51 R3815-8

[97] Ladd A J C 1990 Hydrodynamic transport coefficients of random dispersions of hard spheres J. Chem. Phys. 93 3484-94

[98] Moncho-Jordá A, Louis A A and Padding J T 2010 Effects of interparticle attractions on colloidal sedimentation Phys. Rev. Lett. 104068301

[99] Gilleland W T, Torquato S and Russel W B 2011 New bounds on the sedimentation velocity for hard, charged and adhesive hard-sphere colloids J. Fluid Mech. 667 403-25

[100] Moncho-Jordá A, Louis A A and Padding J T 2012 How Péclet number affects microstructure and transient cluster aggregation in sedimenting colloidal suspensions J. Chem. Phys. 136064517

[101] Buscall R, Goodwin J W, Ottewill R H and Tadros T F 1982 The settling of particles through Newtonian and non-Newtonian media J. Colloid Interface Sci. 85 78-86

[102] Bacri J-C, Frénois C, Hoyos M, Perzynski R, Rakotomalala N and Salin D D 1986 Acoustic study of suspension sedimentation Europhys. Lett. 2 123-8

[103] de Kruif C G, Jansen J W and Vrij A 1987 Sterically stabilized silica colloid as a model supramolecular fluid Physics of Complex and Supermolecular Fluids ed S A Safran and N A Clark (New York: Wiley)

[104] Xue J Z, Herbolzheimer E, Rutgers M A, Russel W B and Chaikin P M 1992 Diffusion, dispersion, and settling of hard spheres Phys. Rev. Lett. 69 1715-8

[105] Buzzaccaro S, Tripodi A, Rusconi R, Vigolo D and Piazza R 2008 Kinetics of sedimentation in colloidal suspensions J. Phys: Condens. Matter 20494219

[106] Esipov S E 1995 Coupled Burger equations: a model of polydispersive sedimentation Phys. Rev. E 52 3711-8

[107] Marini Bettolo Marconi U and Tarazona P 1999 Dynamic density functional theory of fluids J. Chem. Phys. 110 8032-44

[108] Royall C P, Dzubiella J, Schmidt M and van Blaaderen A 2007 Nonequilibrium sedimentation of colloids on the particle scale Phys. Rev. Lett. 98188304

[109] Caflisch R E and Luke J H C 1985 Variance in the sedimentation speed of a suspension Phys. Fluids 28 759-60

[110] Segrè P N, Herbolzheimer E and Chaikin P M 1997 Long-range correlations in sedimentation Phys. Rev. Lett. 79 2574-7

[111] Nicolai H, Herzhaft B, Hinch E J, Oger L and Guazzelli E 1995 Particle velocity fluctuations and hydrodynamic self-diffusion of sedimenting nonBrownian spheres Phys. Fluids 7 12-23

[112] Cowan M L, Page J H and Weitz D A 2000 Velocity fluctuations in fluidized suspensions probed by ultrasonic correlation spectroscopy Phys. Rev. Lett. 85 453-6

[113] Brenner M P 1999 Screening mechanisms in sedimentation Phys. Fluids 11 754-72

[114] Mucha P J, Tee S Y, Weitz D A, Shraiman B I and Brenner M P 2004 A model for velocity fluctuations in sedimentation J. Fluid Mech. 501 71-104

[115] Guazzelli E and Hinch J 2011 Fluctuations and instability in sedimentation Annu. Rev. Fluid Mech. 43 97-116

[116] Koch D L and Shaqfeh E S G 1991 Screening in sedimenting suspensions J. Fluid Mech. 224 275-303

[117] Felderhof B U 2008 Hydrodynamic screening and axisymmetric turbulence in the transient sedimentation of a dilute suspension at small Reynolds number Physica A 387 5999-6012

[118] Lei X, Ackerson B J and Tong P 2001 Settling statistics of hard sphere particles Phys. Rev. Lett. 86 3300-3 
[119] Tee S Y, Mucha P J, Cipelletti L, Manley S, Brenner M P, Segre P N and Weitz D A 2002 Nonuniversal velocity fluctuations of sedimenting particles Phys. Rev. Lett. 89054501

[120] Lee S, Jang Y, Choi C and Lee T 1992 Combined effect of sedimentation velocity fluctuation and selfsharpening on interface broadening Phys. Fluids A 4 2601-6

[121] Bergougnoux L, Ghicini S, Guazzelli E and Hinch J 2003 Spreading fronts and fluctuations in sedimentation Phys. Fluids $\mathbf{1 5}$ 1875-87

[122] Hinch E J Sedimentation of small particles Disorder and Mixing ed E Guyon et al (Dordrecht: Kluwer)

[123] Ladd A J C 2002 Effects of container walls on the velocity fluctuations of sedimenting spheres Phys. Rev. Le\$8 048301

[124] Padding J T and Louis A A 2004 Hydrodynamic and Brownian fluctuations in sedimenting suspensions Phys. Rev. Lett. 93220601

[125] Cipelletti L, Brambilla G, Maccarrone S and Caroff S 2013 Simultaneous measurement of the microscopic dynamics and the mesoscopic displacement field in soft systems by speckle imaging Opt. Express 21 22353-66

[126] Buzzaccaro S, Secchi E and Piazza R 2013 Ghost particle velocimetry: accurate 3D flow visualization using standard lab equipment Phys. Rev. Lett. 111

[127] Da(4ik8KOE, Russel W B and Glantschnig W J 1991 Settling suspensions of colloidal silica: observations and $\mathrm{x}$-ray measurements J. Chem. Soc. Faraday Trans. 87 411-

[128] Ackerson B J, Paulin S E, Johnson B, van Megen W and Underwood S 1999 Crystallization by settling in suspensions of hard spheres Phys. Rev. E 59 6903-

[129] Liblex S R, Borohovich S, Butenko A V, Schofield A B and Sloutskin E 2013 Dense colloidal fluids form denser amorphous sediments Proc. Natl Acad. Sci. USA 110 5769-73

[130] Torquato S and Stillinger F H 2003 Local density fluctuations, hyperuniformity, and order metrics. Phys. Rev. E 68041113

[131] Blanchette F and Bush J W 2005 Particle concentration evolution and sedimentation-induced instabilities in a stably stratified environment Phys. Fluids 17073302

[132] Batchelor G K and Wen C S 1982 Sedimentation in a dilute polydisperse system of interacting spheres:II. Numerical results J. Fluid Mech. 124 495-528

[133] Boycott A E 1920 Sedimentation of blood corpuscles Nature 104532

[134] Heitkam S, Yoshitake Y, Toquet F, Langevin D and Salonen A 2013 Speeding up of sedimentation under confinements Phys. Rev. Lett. 110178302
[135] Piazza R 2008 Thermophoresis: moving particles with thermal gradients Soft Matter 4 1740-4

[136] Bruneau D, Feuillebois F, Bławzdziewicz J and Anthore R 1998 Three-dimensional intrinsic convection in dilute and dense dispersions of settling spheres Phys. Fluids 10 55-9

[137] Foscolo P U, Gibilaro L G and Waldram S P 1983 A unified model for particulate expansion of fluidised beds and flow in fixed porous media Chem. Eng. Sci. 38 1251-60

[138] Gibb J 1991 Pressure and viscous forces in an equilibrium fluidized suspension Chem. Eng. Sci. 46 377-9

[139] Jean R H and Fan L S 1992 On the model equations of Gibilaro and Foscolo with corrected buoyancy force Powder Technol. 42 201-5

[140] Khan A R and Richardson J F 1990 Pressure gradient and friction factor for sedimentation and fluidisation of uniform spheres in liquids Chem. Eng. Sci. 45 255-65

[141] Piazza R, Buzzaccaro S, Secchi E and Parola A 2013 On the general concept of buoyancy in sedimentation and ultracentrifugation Phys. Biol. 10045005

[142] Casassa E F and Eisenberg H 1964 Thermodynamic analysis of multicomponent solutions Adv. Protein Chem. 19 287-395

[143] Eisenberg H 2003 Adair was right in his time Eur. Biophys. J. 32 406-11

[144] Piazza R, Buzzaccaro S, Secchi E and Parola A 2012 What buoyancy really is. A generalized Archimedes' principle for sedimentation and ultracentrifugation Soft Matter $87112-5$

[145] Parola A, Buzzaccaro S, Secchi E and Piazza R 2013 Sedimentation equilibrium and the generalized Archimedes' principle J. Chem. Phys. 138114907

[146] Mansoori G A, Carnahan N F, Starling K E and Leland T W 1971 Equilibrium thermodynamic properties of the mixture of hard spheres J. Chem. Phys. 54 1523-5

[147] Tohver V, Smay J E, Braem A, Braun P V and Lewis J A 2001 Nanoparticle halos: a new colloid stabilization mechanism Proc. Natl Acad. Sci. USA 98 8950-4

[148] Karanikas S and Louis A A 2004 Dynamic colloidal stabilization by nanoparticle halos Phys. Rev. Lett. 93248303

[149] de las Heras D, Doshi N, Cosgrove T, Phipps J, Gittins D I, van Duijneveldt J S and Schmidt M 2012 Floating nematic phase in colloidal platelet-sphere mixtures $S c i$. Rep. 2789

[150] Schmidt M, Dijkstra M and Hansen J-P 2004 Floating liquid phase in sedimenting colloid-polymer mixtures Phys. Rev. Lett. 93088303 